\title{
Disentangling running coupling and conformal effects in QCD
}

\author{
S. J. Brodsky, ${ }^{1}$ E. Gardi, ${ }^{2,3,4}$ G. Grunberg, ${ }^{2}$ and J. Rathsman ${ }^{4}$ \\ ${ }^{1}$ Stanford Linear Accelerator Center, Stanford University, Stanford, California 94309 \\ ${ }^{2}$ Centre de Physique Théorique de l'Ecole Polytechnique, 91128 Palaiseau Cedex, France \\ ${ }^{3}$ Laboratoire de Physique Théorique, Université de Paris XI, 91405 Orsay Cedex, France \\ ${ }^{4}$ TH Division, CERN, CH-1211 Geneva 23, Switzerland*
}

(Received 10 October 2000; published 6 April 2001)

\begin{abstract}
We investigate the relation between a postulated skeleton expansion and the conformal limit of QCD. We begin by developing some consequences of an Abelian-like skeleton expansion, which allows one to disentangle running-coupling effects from the remaining skeleton coefficients. The latter are by construction renormalon free, and hence hopefully better behaved. We consider a simple ansatz for the expansion, where an observable is written as a sum of integrals over the running coupling. We show that in this framework one can set a unique Brodsky-Lepage-Mackenzie (BLM) scale-setting procedure as an approximation to the runningcoupling integrals, where the BLM coefficients coincide with the skeleton ones. Alternatively, the runningcoupling integrals can be approximated using the effective charge method. We discuss the limitations in disentangling running coupling effects in the absence of a diagrammatic construction of the skeleton expansion. Independently of the assumed skeleton structure we show that BLM coefficients coincide with conformal coefficients defined in the small $\beta_{0}$ (Banks-Zaks) limit where a perturbative infrared fixed point is present. This interpretation of the BLM coefficients should explain their previously observed simplicity and smallness. Numerical examples are critically discussed.
\end{abstract}

DOI: 10.1103/PhysRevD.63.094017

PACS number(s): 12.38.Bx

\section{INTRODUCTION}

The large-order behavior of a perturbative expansion in gauge theories is inevitably dominated by the factorial growth of renormalon diagrams [1-4]. In the case of quantum chromodynamics (QCD), the coefficients of perturbative expansions in the QCD coupling $\alpha_{s}$ can increase dramatically even at low orders. This fact, together with the apparent freedom in the choice of renormalization scheme and renormalization scales, limits the predictive power of perturbative calculations, even in applications involving large momentum transfer where $\alpha_{s}$ is effectively small.

A number of theoretical approaches have been developed to reorganize the perturbative expansions in an effort to improve the predictability of perturbative QCD. For example, optimized scale and scheme choices have been proposed, such as the method of effective charges (ECH) [5], the principle of minimal sensitivity (PMS) [6], and the BrodskyLepage-Mackenzie (BLM) scale-setting prescription [7] and its generalizations [8-20]. More recent developments [4] include the resummation of the formally divergent renormalon series and the parametrization of related higher-twist powersuppressed contributions.

In general, a factorially divergent renormalon series arises when one integrates over the logarithmically running coupling $\alpha_{s}\left(k^{2}\right)$ in a loop diagram. Such contributions do not occur in conformally invariant theories which have a constant coupling. Of course, in the physical theory, the QCD coupling does run. Nevertheless, relying on a postulated "dressed skeleton expansion," we shall show that a conformal series is directly relevant to physical QCD predictions.

\footnotetext{
*Present address.
}

In quantum electrodynamics the dressed skeleton expansion can replace the standard perturbative expansion. The skeleton diagrams are defined as those Feynman graphs where the three-point vertex and the lepton and photon propagators have no substructure [21]. Thanks to the QED Ward identity, the renormalization of the vertex cancels against the lepton self-energy, while the effect of dressing the photons in the skeleton diagrams by vacuum polarization insertions can be computed by integrating over the GellMann-Low effective charge $\bar{\alpha}\left(k^{2}\right)$. The perturbative coefficients defined from the skeleton graphs themselves are conformal - they correspond to the series in a theory with a zero $\beta$ function. Therefore they are entirely free of running coupling effects such as renormalons. Each term in the dressed skeleton expansion resums renormalon diagrams to all orders in a renormalization scheme invariant way. The resummation ambiguity, which is associated with scales where the coupling becomes strong, can be resolved only at the non-perturbative level.

In QCD, a skeleton expansion can presumably be constructed based on several different dressed Green functions (see [22]). A much more interesting possibility, which is yet speculative, is the existence of an Abelian-like skeleton expansion, with only one effective charge function. The construction of such an expansion is not straightforward due to the presence of gluon self-interaction diagrams and the essential difference between vacuum polarization insertions and charge renormalization. Nevertheless, at the one-loop level there is a diagrammatic algorithm, the so-called "pinch technique" [23], which allows one to identify in every nonAbelian diagram the part which can be absorbed into the renormalization of the effective gluon propagator. The sum of all the vacuum-polarization-like parts turns out to be gauge invariant, thus defining a natural candidate for the 
non-Abelian equivalent of the Gell-Mann-Low effective charge, $\bar{\alpha}_{s}\left(k^{2}\right)$. Moreover, the pinch technique leads to Ward identities similar to those of the Abelian theory: after the vacuum-polarization-like parts have been taken into account, the remaining vertex correction cancels against the quark self-energy. In this way the pinch technique achieves the first step in the construction of an Abelian-like skeleton expansion. Recently there have been some encouraging developments $[24,25]$ in the application of the pinch technique beyond one loop and its possible relation to the background field method. The hope is that these techniques will eventually provide proof of the existence of the skeleton expansion as well as an all-order constructive definition for the nonAbelian skeleton structure and the non-Abelian skeleton effective charge $\bar{\alpha}_{s}\left(k^{2}\right)$.

In this paper, we shall postulate that an Abelian-like skeleton expansion can be defined at arbitrary order in QCD. We shall not deal here with the diagrammatic construction of the skeleton expansion but rather restrict ourselves to the consequences which follow from such a structure. To this end we will introduce a simple ansatz for the skeleton expansion, where similarly to the Abelian case, a generic observable is written as a sum of integrals over the running coupling. As in QED, we can then identify running coupling effects to all orders, and treat them separately from the conformal part of the perturbative expansion. A considerable simplification is achieved, for instance, by assuming that the dependence on the number of light quark flavors, $N_{f}$, originates only in the running coupling itself, as in Abelian theory with light-bylight diagrams being excluded. As a consequence, the coefficients appearing in the assumed skeleton expansion are $N_{f}$ independent. By construction these skeleton coefficients are free of renormalons and are therefore expected to be better behaved. We will show that they have a simple interpretation in the presence of a perturbative infrared fixed point, as occurs in the small $\beta_{0}$ limit: they are the "conformal" coefficients in the series relating the fixed-point value of the observable under consideration with that of the skeleton effective charge. Thus, given the assumption that these coefficients are $N_{f}$ independent, they can be obtained from standard perturbative coefficients using the Banks-Zaks expansion [26-28], where the fixed-point coupling is expanded in powers of $\beta_{0}$.

The conformal series can be seen as a template $[9,10]$ for physical QCD predictions, where instead of the fixed coupling one has at each order a weighted average of the skeleton effective charge $\bar{\alpha}_{s}\left(k^{2}\right)$ with respect to an observable(and order-) dependent momentum distribution function. The momentum integral corresponding to each skeleton term is renormalization-scheme invariant. Had the skeleton effective charge been known at all scales, this integral could have been unambiguously evaluated, thus including both perturbative and non-perturbative contributions. In practice it can be evaluated up to power-suppressed ambiguities, which originate in the infrared where the coupling becomes strong. These infrared renormalon ambiguities can be resolved only by explicitly taking non-perturbative effects into account. Since such effects cannot be calculated with present meth- ods, they can only be parametrized. Indeed, a natural parametrization in the form of an infrared finite coupling [29] emerges from the structure of the skeleton integral. This way the skeleton expansion gives a natural framework in which renormalon resummation and the analysis of nonperturbative power corrections are performed together $[30,31]$.

As an alternative to computing a dressed skeleton integral, one can approximate it by evaluating the coupling at the BLM scale [7], in analogy to the mean-value theorem [11]. By going to higher orders in the perturbative expansion, this approximation can be systematically improved. Another possibility considered here is to approximate each dressed skeleton term separately using the effective charge approach. This approach is tailored [5] to deal with running coupling effects and it bypasses the remaining scheme and scale setting ambiguities in the power series expressions for the BLM scales. Assuming a simple form of the skeleton expansion, running coupling effects can be disentangled from the remaining conformal expansion by tracing the $N_{f}$ dependence of the coefficients. In this case BLM scale setting (or the ECH alternative) can be applied to a generic QCD observable based on knowledge of the first few coefficients. In the general case disentangling running coupling effects becomes more involved, and it eventually requires a diagrammatic construction of the skeleton expansion. We emphasize that both the BLM scale-setting method and the suggested ECH method remain on the perturbative level and, as opposed to the infrared finite coupling approach mentioned above, these methods are not particularly suited to deal with renormalon ambiguities and the related power corrections.

BLM scale setting can also be applied to the perturbative relation between the effective charges of two physical observables. This results in a specific "commensurate scale relation" [12] between the two quantities. The coefficients appearing in such relations are conformal and, as guaranteed by the transitivity property of the renormalization group, they do not depend on the intermediate scheme used. This way conformal relations appear to be relevant for real-world QCD predictions even in the absence of a complete understanding of the underlying skeleton structure. In the case of the Crewther relation $[32,33,18]$, which connects the effective charges of the $e^{+} e^{-}$annihilation cross section to the Bjorken and Gross-Llewellyn-Smith sum rules for deepinelastic scattering, the conformal relation is simply a geometric series. This example highlights the power of characterizing QCD perturbative expansions in terms of conformal coefficients.

This paper is organized as follows: we begin in Sec. II by recalling the concept of the skeleton expansion in the Abelian case [21] and stating the main assumptions concerning the non-Abelian case. We continue, in Sec. III, by reviewing the standard BLM scale-setting procedure and recalling the ambiguity of the procedure beyond the next-to-leading order. We then show how this ambiguity is resolved upon assuming a skeleton expansion, provided we work in the appropriate renormalization scheme, the "skeleton scheme," and require a one-to-one correspondence between the terms in the BLM series and the dressed skeletons. We also discuss in this sec- 
tion the limitations of applying the formal BLM procedure in the absence of a diagrammatic construction of the skeleton expansion.

In Sec. IV we present an alternative to performing an explicit scale setting, by using the ECH method as a tool to resum running-coupling effects within the framework of the assumed skeleton expansion. Close connections between the two approaches are pointed out. In the Appendix we look at the original ECH approach from the point of view of the skeleton expansion, comparing it to the application of the $\mathrm{ECH}$ method to the leading skeleton term. We also calculate there the $\beta_{0}=0$ limit of the skeleton coupling $\beta$ function coefficient $\bar{\beta}_{2}$.

We then come to the main subject of the paper, the relation between the coefficients which remain after applying BLM scale setting and the conformal limit of QCD. We derive (Sec. V) a relation between these BLM coefficients and the conformal coefficients defined in the infrared limit in the conformal window, where a non-trivial perturbative fixedpoint exists [34-38]. In Sec. VI we show explicitly that the conformal coefficients, calculated using the Banks-Zaks expansion, are the same as the ones in the BLM series. In Sec. VII we recall previous observations concerning the smallness of conformal and Banks-Zaks coefficients, and examine whether this apparent convergence can be explained by the absence of renormalons in such relations. The conclusions are given in Sec. VIII.

\section{RENORMALONS AND THE SKELETON EXPANSION}

Consider a Euclidean QED observable $a_{R}\left(Q^{2}\right)$, which depends on a single external space-like momentum $Q^{2}$ and is normalized as an effective charge. The perturbative expansion in a generic renormalization scheme is then given by

$$
a_{R}\left(Q^{2}\right)=a\left(\mu^{2}\right)+r_{1} a\left(\mu^{2}\right)^{2}+r_{2} a\left(\mu^{2}\right)^{3}+\cdots,
$$

where $a=\alpha / \pi$ and $\mu$ is the renormalization scale.

The perturbative series can be reorganized and written in the form of a skeleton expansion

$$
a_{R}\left(Q^{2}\right)=R_{0}\left(Q^{2}\right)+s_{1} R_{1}\left(Q^{2}\right)+s_{2} R_{2}\left(Q^{2}\right)+\cdots,
$$

where the first term, $R_{0}$, corresponds to a single dressed photon: it is the infinite set of "renormalon diagrams" obtained by all possible vacuum polarization insertions into a single photon line. The second term, $s_{1} R_{1}$, corresponds to a double dressed-photon exchange and so on. In QED, vacuum polarization insertions amount to charge renormalization. Thus $R_{0}$ can be written as

$$
R_{0}\left(Q^{2}\right) \equiv \int_{0}^{\infty} \bar{a}\left(k^{2}\right) \phi_{0}\left(k^{2} / Q^{2}\right) \frac{d k^{2}}{k^{2}}
$$

where $k^{2}$ is the virtuality of the exchanged photon, $\bar{a}\left(k^{2}\right)$ is the Gell-Mann-Low effective charge representing the full propagator, and $\phi_{0}$ is the (observable dependent) Feynman integrand for a single photon exchange diagram, which is interpreted as the photon momentum distribution function [14]. Similarly, $R_{1}$ is given by

$$
R_{1}\left(Q^{2}\right) \equiv \int_{0}^{\infty} \bar{a}\left(k_{1}^{2}\right) \bar{a}\left(k_{2}^{2}\right) \phi_{1}\left(k_{1}^{2} / Q^{2}, k_{2}^{2} / Q^{2}\right) \frac{d k_{1}^{2}}{k_{1}^{2}} \frac{d k_{2}^{2}}{k_{2}^{2}}
$$

and so on.

For convenience the normalization of $\phi_{i}$ in $R_{i}\left(Q^{2}\right)$ has been set to 1 such that the $R_{i}\left(Q^{2}\right)$ in Eq. (2) have an expansion $R_{i}\left(Q^{2}\right)=\bar{a}\left(Q^{2}\right)^{i+1}+\ldots$. For example, the normalization of $\phi_{0}\left(k^{2} / Q^{2}\right)$ in $R_{0}$ is

$$
\int_{0}^{\infty} \phi_{0}\left(k^{2} / Q^{2}\right) \frac{d k^{2}}{k^{2}}=1
$$

In QED fermion loops appear either dressing the exchanged photons or in light-by-light type diagrams, where they are attached to four or more photons (an even number). Barring the latter, the dependence on the number of massless fermion flavors, $N_{f}$, is fully contained in the Gell-Mann-Low effective charge. It follows that the skeleton coefficients $s_{i}$ as well as the momentum distribution functions $\phi_{i}$ are entirely free of $N_{f}$ dependence. Light-by-light type diagrams have to be treated separately, as the starting point of new skeleton structures.

The skeleton expansion (2) is a renormalization group invariant expansion: each term is by itself scheme invariant. This is in contrast with the standard scale and scheme dependent perturbative expansion (1). The renormalons in Eq. (1) can be obtained upon expansion of the dressed skeleton terms in Eq. (2) in some scheme. Let us consider first the leading skeleton (3) and examine, for simplicity, its expansion in $\bar{a}\left(Q^{2}\right)$. We assume that $\bar{a}\left(k^{2}\right)$ obeys the renormalization group equation

$\frac{d \bar{a}\left(k^{2}\right)}{d \ln k^{2}}=-\left[\beta_{0} \bar{a}\left(k^{2}\right)^{2}+\beta_{1} \bar{a}\left(k^{2}\right)^{3}+\bar{\beta}_{2} \bar{a}\left(k^{2}\right)^{4}+\cdots\right] \equiv \bar{\beta}(\bar{a})$

where $\beta_{0}$ is negative in QED and positive in QCD. Then $\bar{a}\left(k^{2}\right)$ can be expanded as

$$
\begin{aligned}
\bar{a}\left(k^{2}\right)= & \bar{a}\left(Q^{2}\right)+\beta_{0} t \bar{a}\left(Q^{2}\right)^{2}+\left(\beta_{1} t+\beta_{0}^{2} t^{2}\right) \bar{a}\left(Q^{2}\right)^{3} \\
& +\left(\bar{\beta}_{2} t+\frac{5}{2} \beta_{1} \beta_{0} t^{2}+\beta_{0}^{3} t^{3}\right) \bar{a}\left(Q^{2}\right)^{4}+\cdots
\end{aligned}
$$

where $t \equiv-\ln \left(k^{2} / Q^{2}\right)$. Inserting this into Eq. (3) under the integration sign we obtain

$$
\begin{aligned}
R_{0}\left(Q^{2}\right)= & \bar{a}\left(Q^{2}\right)+r_{1}^{(1)} \beta_{0} \bar{a}\left(Q^{2}\right)^{2}+\left(r_{2}^{(2)} \beta_{0}^{2}+r_{1}^{(1)} \beta_{1}\right) \bar{a}\left(Q^{2}\right)^{3} \\
& +\left(r_{3}^{(3)} \beta_{0}^{3}+\frac{5}{2} r_{2}^{(2)} \beta_{1} \beta_{0}+r_{1}^{(1)} \bar{\beta}_{2}\right) \bar{a}\left(Q^{2}\right)^{4}+\cdots
\end{aligned}
$$

where 


$$
r_{i}^{(i)} \equiv \int_{0}^{\infty}\left[-\ln \left(k^{2} / Q^{2}\right)\right]^{i} \phi_{0}\left(k^{2} / Q^{2}\right) \frac{d k^{2}}{k^{2}} .
$$

We note that in the large $N_{f}$ (large $\beta_{0}$ ) limit, ${ }^{1}$ the perturbative coefficients $r_{i}=r_{i}^{(i)}$ and thus ${ }^{2}$

$$
\left.a_{R}\left(Q^{2}\right)\right|_{\operatorname{large} \beta_{0}}=\bar{a}\left(Q^{2}\right)\left[\sum_{i=0}^{\infty} r_{i}^{(i)}\left[\beta_{0} \bar{a}\left(Q^{2}\right)\right]^{i}+\mathcal{O}\left(1 / \beta_{0}\right)\right] .
$$

At large orders $i \gg 1$, both small and large momentum regions become dominant in Eq. (9), giving rise to the characteristic renormalon factorial divergence $\left(r_{i}^{(i)} \sim i\right.$ !). As mentioned above, this is believed to be the dominant source of divergence of the perturbative expansion (1). On the other hand, in the skeleton expansion (2) the renormalons are by definition resummed and so the remaining coefficients $s_{i}$ should be free of this divergence. These coefficients are expected to increase much slower leading to a better behaved expansion.

As mentioned in the Introduction, the generalization of the Abelian skeleton expansion to QCD is not straightforward. Diagrammatically, the skeleton expansion in QCD has a simple realization only in the large $N_{f}$ limit where gluon self-interaction contributions are negligible so that the theory resembles QED. ${ }^{3}$ In the framework of renormalon calculus, one returns from the large $N_{f}$ limit to real world QCD by replacing $N_{f}$ with the linear combination of $N_{f}$ and $C_{A}$ $=N_{c}$ which appears in the leading coefficient [40] of the $\beta$ function:

$$
\beta_{0}=\frac{1}{4}\left(\frac{11}{3} C_{A}-\frac{2}{3} N_{f}\right)
$$

This replacement, usually called "naive nonAbelianization" [41,14-16], amounts to taking into account a gauge invariant set of diagrams which is responsible for the one-loop running of the coupling constant.

To go beyond the "naive non-Abelianization" level constructing an Abelian-like skeleton expansion in QCD, one

\footnotetext{
${ }^{1}$ In QCD, the Abelian correspondence in the large $N_{f}$ limit requires that the coefficient $\bar{\beta}_{i}$ of the skeleton coupling $\beta$ function (6) not contain $N_{f}^{i+1}$. It has to be a polynomial of order $N_{f}^{i}$ in $N_{f}$. This would guarantee that in the large $N_{f}$ limit $\bar{\beta}(\bar{a})$ is just the one-loop $\beta$ function. Note that while some schemes [e.g. the modified minimal subtraction scheme $(\overline{\mathrm{MS}})$ and static potential effective charge] have this property, generic effective charges (defined through observable quantities) do not. This property of the skeleton scheme is used making the identification of $r_{i}^{(i)}$ in Eq. (8) as the large $N_{f}$ coefficients.

${ }^{2} \mathrm{We}$ comment that the sub-leading terms in $1 / N_{f}$ in Eq. (8) of the form $\beta_{1} \beta_{0}^{i-2}$ were computed to all orders in [15]. However, other terms which involve higher order coefficients of the $\beta$ function contribute at the same level in $1 / N_{f}$.

${ }^{3}$ This can also be understood from the $C_{A} \rightarrow 0$ limit discussed in Ref. [39].
}

needs a method to identify skeleton structures and to isolate vacuum-polarization-like insertions which are responsible for the running of the coupling at any order. The pinch technique [23-25] may provide a systematic way to make this identification. The resulting set of skeleton structures would surely be larger than in the Abelian theory. It may include, for example, fermion loops attached to an odd number of gluons, which vanish in the Abelian limit. Like Abelian light-by-light type diagrams, these structures should be treated separately. As opposed to Abelian theory, where light-by-light type diagrams are distinguished by their characteristic dependence on the charges, in the non-Abelian case these structures may not be separable based only on their color group structure. We assume that there is a unique (gauge invariant) way to identify skeleton structures in QCD, making Eq. (2) relevant. In much of the discussion that follows we shall further make the assumption that the entire dependence of $a_{R}\left(Q^{2}\right)$ on $N_{f}$ is through the running coupling. Thus in our ansatz $s_{i}$ and $\phi_{i}$ are $N_{f}$ independent, just like in the Abelian case with light-by-light diagrams being excluded. Of course, the class of diagrams containing fermion loops as part of the skeleton structure should eventually be taken into account.

Another simplifying assumption we made already in the Abelian case is that our ansatz (2) contains only one skeleton at each order, whereas in general there will be several skeletons contributing at each order. The simplest example is $e^{-} e^{-}$scattering with both $t$ - and $u$-channel exchange. Several skeletons at the same order also occur in single-scale observables considered here, and therefore Eq. (2) should be generalized accordingly. We shall return to this point in the next section.

We stress that the coupling constant $\bar{a}\left(k^{2}\right)$ in Eq. (3) is understood to be a specific effective charge, in analogy to the Gell-Mann-Low effective charge in QED. This "skeleton effective charge" $\bar{a}\left(k^{2}\right)$ should be defined diagrammatically order by order in perturbation theory. In the framework of the pinch technique, $\bar{a}\left(k^{2}\right)$ has been identified at the oneloop level, ${ }^{4}$ e.g. it is related to the $\overline{\mathrm{MS}}$ coupling by

$$
\begin{aligned}
\bar{a}\left(k^{2}\right)= & a \overline{\mathrm{MS}}\left(\mu^{2}\right)+\left[-\beta_{0}\left(\log \frac{k^{2}}{\mu^{2}}-\frac{5}{3}\right)+\frac{C_{A}}{3}\right] a_{\overline{\mathrm{MS}}}\left(\mu^{2}\right)^{2} \\
& +\cdots .
\end{aligned}
$$

Recently, there have been encouraging developments [25,24] in the application of the pinch technique beyond one loop. This would hopefully lead to a systematic identification of the "skeleton effective charge", at higher orders, namely the determination of higher order coefficients $\left(\bar{\beta}_{i}\right.$ for $\left.i \geqslant 2\right)$ of the $\beta$ function $\bar{\beta}(\bar{a})=d \bar{a} / d \ln k^{2}$. This $\beta$ function should coincide with the Gell-Mann-Low function upon taking the Abelian limit $C_{A}=0$ (see Ref. [39]).

Being scheme invariant and free of renormalon divergence, the skeleton expansion (2) seems much favorable over

\footnotetext{
${ }^{4}$ This means that the corresponding QCD scale $\bar{\Lambda}$ is identified.
} 
the standard perturbative QCD expansion (1). This advantage may become crucial in certain applications, e.g. for the extraction of $\alpha_{s}$ from event shape variables [31]. However, in the absence of a concrete all-order diagrammatic definition for the skeleton expansion in QCD, running-coupling effects cannot be systematically resummed beyond the single dressed gluon level corresponding to the leading skeleton. In particular, the momentum distribution functions of the subleading skeletons are not known. On the other hand, the BLM scale-setting procedure, which is well defined at higher orders, can be considered as a manifestation of the skeleton expansion. As we shall see, it is possible in this framework to approximate the sub-leading skeleton terms, provided the correct skeleton scheme is used. Currently, since the skeleton effective charge has not been identified, the choice of scheme in the BLM procedure remains an additional essential ingredient.

\section{BLM SCALE SETTING}

The BLM approach [7] is motivated by the skeleton expansion. The basic idea is that the dressed skeleton integral (3) can be well approximated by $R_{0} \simeq \bar{a}\left(\mu^{2}\right)+\cdots$ provided that the renormalization scale $\mu$ is properly chosen. Indeed, by the mean value theorem [11], there exists a scale $k_{0}$ such that

$$
\begin{aligned}
R_{0}\left(Q^{2}\right) & =\int_{0}^{\infty} \bar{a}\left(k^{2}\right) \phi_{0}\left(k^{2} / Q^{2}\right) \frac{d k^{2}}{k^{2}} \\
& =\bar{a}\left(k_{0}^{2}\right) \int_{0}^{\infty} \phi_{0}\left(k^{2} / Q^{2}\right) \frac{d k^{2}}{k^{2}}=\bar{a}\left(k_{0}^{2}\right)
\end{aligned}
$$

where the last step follows from the assumed normalization for $\phi_{i}$ Eq. (5).

A first approximation to $k_{0}$ is given by the average virtuality of the exchanged gluon:

$$
\begin{aligned}
k_{0,0}^{2} & =Q^{2} \exp \left(\int_{0}^{\infty} \ln \frac{k^{2}}{Q^{2}} \phi_{0}\left(k^{2} / Q^{2}\right) \frac{d k^{2}}{k^{2}} / \int_{0}^{\infty} \phi_{0}\left(k^{2} / Q^{2}\right) \frac{d k^{2}}{k^{2}}\right) \\
& =Q^{2} \exp \left(-r_{1}^{(1)}\right)
\end{aligned}
$$

where $r_{1}^{(1)}$ is the next-to-leading coefficient of $a_{R}$ in the large $\beta_{0}$ limit (9). The scale (14) is called the "leading order BLM scale." It can be determined directly from the $N_{f}$ dependent part of the next-to-leading coefficient $\left(r_{1}\right)$ in the perturbative series of the observable in terms of $\bar{a}\left(Q^{2}\right)$ :

$$
a_{R}\left(Q^{2}\right)=\bar{a}\left(Q^{2}\right)+r_{1} \bar{a}\left(Q^{2}\right)^{2}+r_{2} \bar{a}\left(Q^{2}\right)^{3}+\cdots
$$

Thanks to the linear $N_{f}$ dependence of $r_{1}$, it can be uniquely decomposed into a term linear in $\beta_{0}$, which is related to the leading skeleton, and a free term

$$
r_{1}=r_{1}^{(0)}+r_{1}^{(1)} \beta_{0}
$$

where both $r_{1}^{(1)}$ and $r_{1}^{(0)}$ are $N_{f}$ independent. After BLM scale setting, with $k_{0,0}^{2}$ given by Eq. (14), one has

$$
a_{R}\left(Q^{2}\right)=\bar{a}\left(k_{0,0}^{2}\right)+r_{1}^{(0)} \bar{a}\left(k_{0,0}^{2}\right)^{2}+\cdots
$$

Thus, technically, the BLM scale-setting procedure amounts, at leading order, to eliminating the $\beta_{0}$ dependent part from the next-to-leading order coefficient. Note that although the leading order BLM scale $k_{0,0}$ of Eq. (14) has a precise meaning as the average gluon virtuality, it is just the lowest order approximation to $k_{0}$ of Eq. (13). In other words, aiming at the evaluation of the leading skeleton term (3), setting the scale as $k_{0,0}$ is just the first step. This approximation can be systematically improved [see Eq. (33) below] in higher orders.

\section{A. Multi-scale BLM and skeleton expansion correspondence}

A BLM series [12] can be written, up to arbitrary high order,

$$
a_{R}\left(Q^{2}\right)=a\left(k_{0}^{2}\right)+c_{1} a\left(k_{1}^{2}\right)^{2}+c_{2} a\left(k_{2}^{2}\right)^{3}+c_{3} a\left(k_{3}^{2}\right)^{4}+\ldots
$$

where the $k_{i}^{2}$ are, in general, different scales proportional to the external scale $Q^{2}$ [as in Eq. (14)] and $c_{i}$ are $N_{f}$ independent coefficients. The intuition behind this generalization is that each skeleton term in Eq. (2) is approximated by a corresponding term in the multi-scale BLM series: each skeleton term may have different characteristic momenta. This one-to-one correspondence with the skeleton expansion requires that the coupling $a$ will be the skeleton effective charge $a=\bar{a}$ such that

$$
R_{i}\left(Q^{2}\right) \equiv \bar{a}\left(k_{i}^{2}\right)^{i+1}
$$

In this case the coefficients of sub-leading terms in Eq. (18) should coincide with the coefficients of the sub-leading skeleton terms, namely $c_{i}=s_{i}$.

More generally, a BLM series can be formally written in an arbitrary scheme: then the coupling $a$ in Eq. (18) can be either defined in a standard scheme like $\overline{\mathrm{MS}}$ or, as suggested in [12], be another measurable effective charge. However, in such cases there is no direct correspondence with the skeleton expansion (2), and as a result the forthcoming motivation for a unique scale setting is lost.

Let us recall how the BLM scale-setting procedure is performed beyond the next-to-leading order [12,8], yielding an expansion of the form (18). Suppose that the perturbative expansion of $a_{R}\left(Q^{2}\right)$ in terms of $a\left(Q^{2}\right)$ is given by ${ }^{5}$

\footnotetext{
${ }^{5}$ We work now in a generic scheme but in contrast to Eq. (1) we start here with the renormalization scale $\mu=Q$, thereby simplifying the formulas that follow. Since the scale is tuned in the BLM procedure, this initial choice is of little significance. The only place where the arbitrary renormalization scale is left at the end is in the power series for the scales shifts, Eq. (23) below.
} 


$$
a_{R}\left(Q^{2}\right)=a\left(Q^{2}\right)+r_{1} a\left(Q^{2}\right)^{2}+r_{2} a\left(Q^{2}\right)^{3}+r_{3} a\left(Q^{2}\right)^{4}+\cdots .
$$

Based on the fact that $r_{i}$ are polynomials of order $i$ in $N_{f}$ and that $\beta_{0}$ and $\beta_{1}$ are linear in $N_{f}$, we can write $r_{1}$ as in Eq. (16) and

$$
r_{2}=r_{2}^{(0)}+r_{2}^{(1)} r_{1}^{(0)} \beta_{0}+r_{2}^{(2)} \beta_{0}^{2}+r_{1}^{(1)} \beta_{1}
$$

where $r_{i}^{(j)}$ are $N_{f}$ independent. The reason for the $\beta_{1}$ dependent term in Eq. (21) shall become clear below. Expanding $a\left(k_{i}^{2}\right)$ in terms of $a\left(Q^{2}\right)$ similarly to Eq. (7), the next-tonext-to-leading order BLM series (18) can be written as

$$
\begin{aligned}
a_{R}\left(Q^{2}\right)= & a\left(Q^{2}\right)+\left(c_{1}+t_{0} \beta_{0}\right) a\left(Q^{2}\right)^{2} \\
& +\left(c_{2}+2 t_{1} c_{1} \beta_{0}+t_{0} \beta_{1}+t_{0}^{2} \beta_{0}^{2}\right) a\left(Q^{2}\right)^{3} .
\end{aligned}
$$

Writing the scale shifts $t_{i} \equiv \ln \left(Q^{2} / k_{i}^{2}\right)$ as a power series in the coupling

$$
t_{i} \equiv t_{i, 0}+t_{i, 1} a\left(Q^{2}\right)+t_{i, 2} a\left(Q^{2}\right)^{2}+\cdots
$$

where $t_{i, 0}$ are assumed to be $N_{f}$ independent, we get

$$
\begin{aligned}
a_{R}\left(Q^{2}\right)= & a\left(Q^{2}\right)+\left(c_{1}+t_{0,0} \beta_{0}\right) a\left(Q^{2}\right)^{2} \\
& +\left[c_{2}+\left(2 t_{1,0} c_{1}+t_{0,1}\right) \beta_{0}+t_{0,0} \beta_{1}+t_{0,0}^{2} \beta_{0}^{2}\right] a\left(Q^{2}\right)^{3} .
\end{aligned}
$$

An order by order comparison of Eqs. (24) and (20) yields the scale shifts $t_{0}=\ln \left(Q^{2} / k_{0}^{2}\right)$ and $t_{1}=\ln \left(Q^{2} / k_{1}^{2}\right)$ and the coefficients $c_{1}$ and $c_{2}$ in terms of $r_{1}$ and $r_{2}$ and the coefficients of the $\beta$ function of $a\left(Q^{2}\right)$. The comparison at the next-toleading order gives

$$
c_{1}=r_{1}^{(0)}
$$

and

$$
t_{0,0}=r_{1}^{(1)} .
$$

The comparison at the next-to-next-to-leading order for the $\beta_{i}$ independent piece gives

$$
c_{2}=r_{2}^{(0)}
$$

while for the $\beta_{0}$ dependent piece it gives

$$
t_{0,1}+2 t_{1,0} r_{1}^{(0)}+\beta_{0}\left(r_{1}^{(1)}\right)^{2}=r_{2}^{(1)} r_{1}^{(0)}+\beta_{0} r_{2}^{(2)} .
$$

Thanks to the explicit $\beta_{1}$ dependent term introduced in Eq. (21), the equality of the corresponding piece there to that in Eq. (24) is satisfied based on the next-to-leading order result (26). To proceed we need to specify $t_{0,1}$ and $t_{1,0}$ such that Eq. (28) is satisfied. Having two free parameters with just one constraint there is apparently no unique solution. Two natural possibilities are the so called multi-scale BLM prescription [12],

$$
t_{0,1}=\beta_{0}\left[r_{2}^{(2)}-\left(r_{1}^{(1)}\right)^{2}\right],
$$

$$
t_{1,0}=\frac{1}{2} r_{2}^{(1)},
$$

and the single-scale BLM prescription [8] where $t_{1,0} \equiv t_{0,0}$ and

$$
t_{0,1}=\beta_{0}\left[r_{2}^{(2)}-\left(r_{1}^{(1)}\right)^{2}\right]-2 r_{1}^{(1)} r_{1}^{(0)}+r_{2}^{(1)} r_{1}^{(0)} .
$$

Having in mind the original motivation for the BLM prescription, it is interesting to examine the case where the scheme of $a$ coincides with the skeleton effective charge $\bar{a}$. Then we would like to have a one-to-one correspondence (19) between the terms in the BLM series (18) and those of the skeleton expansion (2). The multi-scale procedure is consistent with this requirement: the leading term $\bar{a}\left(k_{0}^{2}\right)$ in the BLM series (18) represents only the leading skeleton term $R_{0}$ in Eq. (2), since the scale shift

$$
t_{0}=r_{1}^{(1)}+\left[r_{2}^{(2)}-\left(r_{1}^{(1)}\right)^{2}\right] \beta_{0} \bar{a}\left(Q^{2}\right)
$$

involves only coefficients which are leading in the large $\beta_{0}$ limit and originate in $\phi_{0}$ [cf. Eq. (9)]. On the other hand the single-scale procedure violates this requirement, since there $t_{0}$ involves terms which are sub-leading in $\beta_{0}$ and $d o$ not belong to the leading skeleton term $R_{0}$. In fact, in order to guarantee that the scale shift $t_{0}$ would represent just the leading skeleton $R_{0}$ we are bound to choose $t_{0,1}$ proportional to $\beta_{0}$ and thus the solution (29) is uniquely determined.

We see that a unique scale-setting procedure at the nextto-next-to-leading order $\left(r_{2}\right)$ is implied by the requirement that the scale shift $t_{0}$ should represent the leading skeleton $R_{0}$. In order to continue and apply the BLM prescription at the next order $\left(r_{3}\right)$ we have to impose further constraints based on the structure of both $R_{0}$ and $R_{1}$.

\section{B. BLM scale setting for the leading skeleton}

Let us first examine the structure of the scale shift $t_{0}$ by applying the BLM prescription to a hypothetical observable that contains only an $R_{0}$ term of the form (3). Expanding the coupling $\bar{a}\left(k^{2}\right)$ under the integration sign in terms of $a\left(Q^{2}\right)$ we obtain Eq. (8). We would like to apply the BLM prescription to the latter series, obtaining simply $\bar{a}\left(k_{0}^{2}\right)$, with $t_{0}$ $\equiv \ln \left(Q^{2} / k_{0}^{2}\right)=t_{0,0}+t_{0,1} \bar{a}\left(Q^{2}\right)+\cdots$. Expanding $\bar{a}\left(k_{0}^{2}\right)$ we obtain, from Eq. (7),

$$
\begin{aligned}
\bar{a}\left(k_{0}^{2}\right)= & \bar{a}\left(Q^{2}\right)+\beta_{0} t_{0,0} \bar{a}\left(Q^{2}\right)^{2}+\left(\beta_{0} t_{0,1}+\beta_{1} t_{0,0}\right. \\
& \left.+\beta_{0}^{2} t_{0,0}^{2}\right) \bar{a}\left(Q^{2}\right)^{3}+\left(\beta_{0} t_{0,2}+\beta_{1} t_{0,1}+2 \beta_{0}^{2} t_{0,0} t_{0,1}\right. \\
& \left.+\bar{\beta}_{2} t_{0,0}+\beta_{0}^{3} t_{0,0}^{3}+\frac{5}{2} \beta_{0} \beta_{1} t_{0,0}^{2}\right) \bar{a}\left(Q^{2}\right)^{4}+\cdots
\end{aligned}
$$

Comparing Eq. (8) with Eq. (32) we get 


$$
\begin{aligned}
t_{0}= & r_{1}^{(1)}+\left[r_{2}^{(2)}-\left(r_{1}^{(1)}\right)^{2}\right] \beta_{0} \bar{a}\left(Q^{2}\right) \\
& +\left\{\left[r_{3}^{(3)}-2 r_{1}^{(1)} r_{2}^{(2)}+\left(r_{1}^{(1)}\right)^{3}\right] \beta_{0}^{2}\right. \\
& \left.+\frac{3}{2}\left[r_{2}^{(2)}-\left(r_{1}^{(1)}\right)^{2}\right] \beta_{1}\right\} \bar{a}\left(Q^{2}\right)^{2}+\cdots
\end{aligned}
$$

Here we recovered the two leading orders in $t_{0}$ of Eq. (31). At order $\bar{a}\left(Q^{2}\right)^{2}$ we obtained an explicit dependence on both $\beta_{0}$ and $\beta_{1}$. The combination $r_{2}^{(2)}-\left(r_{1}^{(1)}\right)^{2}$ appearing at the next-to-leading order in $t_{0}$ has an interpretation as the width of the distribution $\phi_{0}$, assuming the latter is positive definite (see $[14,17])$. In general, Eq. (33) can be written in terms of central moments of the distribution $\phi_{0}$, defined by

$$
M_{n}=\left\langle\left(\ln \frac{Q^{2}}{k^{2}}-\left\langle\ln \frac{Q^{2}}{k^{2}}\right\rangle_{\phi_{0}}\right)^{n}\right\rangle_{\phi_{0}}=\left\langle\left(\ln \frac{k_{0,0}^{2}}{k^{2}}\right)^{n}\right\rangle_{\phi_{0}}
$$

for $n \geqslant 2$, where $M_{1}=\left\langle\ln \left(Q^{2} / k^{2}\right)\right\rangle_{\phi_{0}}=\ln \left(Q^{2} / k_{0,0}^{2}\right) \quad$ corresponds to $r_{1}^{(1)}$ in Eq. (9). In terms of the central moments we have

$$
\begin{aligned}
t_{0}= & M_{1}+M_{2} \beta_{0} \bar{a}\left(Q^{2}\right) \\
& +\left\{\left[M_{3}+M_{1} M_{2}\right] \beta_{0}^{2}+\frac{3}{2} M_{2} \beta_{1}\right\} \bar{a}\left(Q^{2}\right)^{2}+\cdots \\
= & M_{1}+M_{2} \beta_{0} \bar{a}\left(k_{0,0}^{2}\right) \\
& +\left\{M_{3} \beta_{0}^{2}+\frac{3}{2} M_{2} \beta_{1}\right\} \bar{a}\left(k_{0,0}^{2}\right)^{2}+\cdots
\end{aligned}
$$

where in the second step we changed the scale from $Q^{2}$ to the leading order BLM scale $k_{0,0}^{2}$ to get simpler expressions for the coefficients of the $t_{0}$ series. At large orders $n$ the moments $M_{n}$ become sensitive to extremely large and small momenta and thus develop renormalon factorial divergence, similarly to the standard perturbative coefficients in Eq. (9). We thus see that in the BLM approach, the scale shift itself is an asymptotic expansion, affected by renormalons.

\section{BLM scale setting for sub-leading skeletons}

Next, let us consider an $R_{1}$ term, given by Eq. (4). Expanding the couplings $\bar{a}\left(k_{1}^{2}\right)$ and $\bar{a}\left(k_{2}^{2}\right)$ under the integral in terms of $\bar{a}\left(Q^{2}\right)$ using Eq. (7), we get [cf. the expansion of $R_{0}$ in Eq. (8)]

$$
\begin{aligned}
R_{1}\left(Q^{2}\right)= & \bar{a}\left(Q^{2}\right)^{2}+\beta_{0} r_{2}^{(1)} \bar{a}\left(Q^{2}\right)^{3}+\left(r_{3}^{(2)} \beta_{0}^{2}+r_{2}^{(1)} \beta_{1}\right) \bar{a}\left(Q^{2}\right)^{4} \\
& +\cdots
\end{aligned}
$$

where

$$
r_{2}^{(1)} \equiv \phi_{1}^{(1,0)}+\phi_{1}^{(0,1)}
$$

$$
r_{3}^{(2)} \equiv \phi_{1}^{(2,0)}+\phi_{1}^{(1,1)}+\phi_{1}^{(0,2)}
$$

with

$$
\begin{aligned}
\phi_{1}^{(j, k)} \equiv & \int_{0}^{\infty}\left[-\ln \left(k_{1}^{2} / Q^{2}\right)\right]^{j} \\
& \times\left[-\ln \left(k_{2}^{2} / Q^{2}\right)\right]^{k} \phi_{1}\left(k_{1}^{2} / Q^{2}, k_{2}^{2} / Q^{2}\right) \frac{d k_{1}^{2}}{k_{1}^{2}} \frac{d k_{2}^{2}}{k_{2}^{2}} .
\end{aligned}
$$

The BLM scale-setting procedure can now be applied according to Eq. (19): $R_{1}\left(Q^{2}\right)$ given in Eq. (36) should be written as $\bar{a}\left(k_{1}^{2}\right)^{2}$. Expanding $\bar{a}\left(k_{1}^{2}\right)^{2}$ in terms of $\bar{a}\left(Q^{2}\right)$ using Eq. (7) and $t_{1}=t_{1,0}+t_{1,1} \bar{a}\left(Q^{2}\right)+\cdots$ we have

$$
\begin{aligned}
\bar{a}\left(k_{1}^{2}\right)^{2}= & \bar{a}\left(Q^{2}\right)^{2}+2 t_{1,0} \beta_{0} \bar{a}\left(Q^{2}\right)^{3}+\left(2 t_{1,1} \beta_{0}+3 t_{1,0}^{2} \beta_{0}^{2}\right. \\
& \left.+2 t_{1,0} \beta_{1}\right) \bar{a}\left(Q^{2}\right)^{4}+\cdots .
\end{aligned}
$$

Comparison with Eq. (36) at next-to-leading order implies

$$
t_{1,0}=\frac{1}{2} r_{2}^{(1)} \text {. }
$$

Comparison at next-to-next-to-leading order then yields

$$
2 t_{1,1} \beta_{0}+\frac{3}{4}\left(r_{2}^{(1)}\right)^{2} \beta_{0}^{2}+r_{2}^{(1)} \beta_{1}=r_{3}^{(2)} \beta_{0}^{2}+r_{2}^{(1)} \beta_{1}
$$

which implies that $t_{1,1}$, just as $t_{0,1}$, is bound to be proportional to $\beta_{0}$. Finally we obtain the scale shift for $R_{1}$ :

$$
t_{1}=\frac{1}{2} r_{2}^{(1)}+\frac{1}{2}\left[r_{3}^{(2)}-\frac{3}{4}\left(r_{2}^{(1)}\right)^{2}\right] \beta_{0} \bar{a}\left(Q^{2}\right) .
$$

Similarly, applying the BLM prescription to $R_{2}$,

$$
R_{2}=\bar{a}\left(Q^{2}\right)^{3}+r_{3}^{(1)} \beta_{0} \bar{a}\left(Q^{2}\right)^{4}+\cdots,
$$

we get

$$
t_{2}=\frac{1}{3} r_{3}^{(1)}
$$

\section{Skeleton decomposition and its limitations}

Let us now return to the case of a generic observable (20) and see that with these skeleton-expansion-correspondence constraints there is a unique BLM scale-setting procedure. The basic idea is that, given the existence of a skeleton expansion, it is possible to separate the entire series into terms which originate in specific skeleton terms. This corresponds to a specific decomposition of each perturbative coefficient $r_{i}$ similarly to Eqs. (16) and (21). Then application of the BLM prescription to the separate skeleton terms, namely representing $R_{i}$ by $\bar{a}\left(k_{i}^{2}\right)^{i+1}$, immediately implies a specific BLM scale-setting procedure for the observable. For example, 
when this procedure is applied up to order $\bar{a}\left(Q^{2}\right)^{4}$, the scale shifts $t_{i}$ for $i=0,1,2$ are given by Eqs. (33), (42) and (44), respectively.

To demonstrate this argument let us simply add up the expanded form of the skeleton terms up to order $\bar{a}\left(Q^{2}\right)^{4}$ with $R_{0}$ given by Eq. (8), $R_{1}$ by Eq. (36) and $R_{2}$ by Eq. (43). For $R_{3}$ we simply have at this order $R_{3}=\bar{a}\left(Q^{2}\right)^{4}$. Altogether we obtain

$$
\begin{aligned}
a_{R}= & \bar{a}+\left[s_{1}+r_{1}^{(1)} \beta_{0}\right] \bar{a}^{2}+\left[s_{2}+s_{1} r_{2}^{(1)} \beta_{0}+r_{2}^{(2)} \beta_{0}^{2}+r_{1}^{(1)} \beta_{1}\right] \bar{a}^{3} \\
& +\left[s_{3}+s_{2} r_{3}^{(1)} \beta_{0}+s_{1} r_{3}^{(2)} \beta_{0}^{2}+r_{3}^{(3)} \beta_{0}^{3}+r_{1}^{(1)} \bar{\beta}_{2}\right. \\
& \left.+\frac{5}{2} r_{2}^{(2)} \beta_{1} \beta_{0}+s_{1} r_{2}^{(1)} \beta_{1}\right] \bar{a}^{4}
\end{aligned}
$$

Here we identify the notation $s_{i}$ which is the coefficient in front of the skeleton term $R_{i}$ with $r_{i}^{(0)}$. We recognize the form of $r_{1}$ and $r_{2}$ as the decompositions introduced before in Eqs. (16) and (21) in order to facilitate application of the BLM prescription. We see that the skeleton expansion structure implies a specific decomposition. Suppose for example we know $r_{1}$ through $r_{3}$ in the skeleton scheme. Equation (45) then defines a unique way to decompose them so that each term corresponds specifically to a given term in the skeleton expansion. The decomposition of $r_{i}$ includes a polynomial in $\beta_{0}$ up to order $\beta_{0}^{i}$ :

$$
s_{i}+\sum_{k=1}^{i} s_{i-k} r_{i}^{(k)} \beta_{0}^{k}
$$

where $s_{0}=1$ by the assumed normalization. The other terms in $r_{i}$ in Eq. (45) depend explicitly on higher coefficients of the $\beta$ function $\bar{\beta}_{j}$ with $1 \leqslant j \leqslant i-1$. Up to order $\bar{a}\left(Q^{2}\right)^{4}$ these terms depend exclusively ${ }^{6}$ on the coefficients $r_{j}^{(k)}$ which appeared at previous orders in the $\beta_{0}$ polynomials (46). Finally, we need to verify that a decomposition of the form (45) is indeed possible. For a generic observable $a_{R}$, the coefficient $r_{i}$ is a polynomial of order $i$ in $N_{f}$. Since the $\beta$ function coefficients $\bar{\beta}_{i}$ are also polynomials of maximal order $i$, the decomposition of $r_{i}$ according to Eq. (45) amounts to solving $i+1$ equations with $i+1$ unknowns: $r_{i}^{(k)}$ with $0 \leqslant k<i$. Thus in general there is a unique solution.

We see that based on the assumed skeleton structure, one can uniquely perform a "skeleton decomposition" and thus also BLM scale setting which satisfies a one-to-one correspondence of the form (19) with the skeleton terms. By construction in this procedure the scale $t_{0}$ is determined exclusively by the large $\beta_{0}$ terms $r_{i}^{(i)}$ which belong to $R_{0}$ [see Eq.

\footnotetext{
${ }^{6}$ As we shall see below, this is no longer true beyond this order, where the coefficients depend on moments which appeared at previous orders, but cannot be expressed in terms of the lower order coefficients themselves.
}

(33)], $t_{1}$ is determined by $r_{i}^{(i-1)}$ terms which belong to $R_{1}$ [see Eq. (42)], $t_{2}$ is determined by $r_{i}^{(i-2)}$ terms which belong to $R_{2}$, etc.

It should be stressed that formally the decomposition (45), and thus also BLM scale setting, can be performed in any scheme: given the coefficients $r_{i}$ up to order $n$, all the coefficients $s_{i}$ and $r_{i}^{(j)}$ for $i \leqslant n$ and $j \leqslant i$ are uniquely determined. No special properties of the "skeleton effective charge", were necessary to show that the decomposition is possible. Even the assumption that for this effective charge the $\beta$ function coefficients $\bar{\beta}_{i}$ are polynomials of order $i$ can be relaxed. For example, the decomposition (45) can be formally performed in physical schemes where $\bar{\beta}_{i}$ are polynomials of order $i+1$. In this case, however, the interpretation of $r_{i}^{(j)}$ in terms of the logarithmic moments of distribution functions is not straightforward. It is also clear that a one-toone correspondence between the BLM prescription and the skeleton expansion (19) exists only if the coupling $a$ is chosen as the skeleton effective charge $\bar{a}$.

Let us now address several complications that limit the applicability of the above discussion. First, we recall the assumption we made that the entire dependence of the perturbative coefficients on $N_{f}$ is related to the running coupling. This means that any explicit $N_{f}$ dependence which is part of the skeleton structure is excluded from Eq. (45). In reality there may be skeletons with fermion loops as part of the structure, which would have to be identified and treated separately.

Having excluded such $N_{f}$ dependence, we have seen that up to order $\bar{a}\left(Q^{2}\right)^{4}$ a formal "skeleton decomposition", (45) of the perturbative coefficients can be performed algebraically without further diagrammatic identification of the skeleton structure. This is no longer true at order $\bar{a}\left(Q^{2}\right)^{5}$, where the "skeleton decomposition" requires the moments of the momentum distribution functions to be identified separately. Such an identification depends on a diagrammatic understanding of the skeleton structure. Looking at $R_{1}$, the coefficient of $\bar{a}\left(Q^{2}\right)^{5}$ in Eq. (36) is

$$
\begin{aligned}
\beta_{0}^{3}[ & \left.\phi_{1}^{(3,0)}+\phi_{1}^{(0,3)}+\phi_{1}^{(1,2)}+\phi_{1}^{(2,1)}\right] \\
& +\beta_{1} \beta_{0}\left[2 \phi_{1}^{(1,1)}+\frac{5}{2}\left(\phi_{1}^{(2,0)}+\phi_{1}^{(0,2)}\right)\right] \\
& +\beta_{2}\left[\phi_{1}^{(1,0)}+\phi_{1}^{(0,1)}\right] .
\end{aligned}
$$

Writing the $\bar{a}\left(Q^{2}\right)^{5}$ term in Eq. (45), one will find, as before, that the terms which depend explicitly on higher coefficients of the $\beta$ function $\bar{\beta}_{l}$, with $1 \leqslant l \leqslant 3$, contain only moments of the skeleton momentum distribution functions $\phi_{i}^{(j, k)}$ which appeared in the decomposition (45) in the coefficients of $\beta_{0}^{j+k} \bar{a}^{1+i+j+k}$ at the previous orders. However, the coefficient of $\beta_{1} \beta_{0}$ will depend on a new linear combination of moments, different from the one identified at order $\bar{a}\left(Q^{2}\right)^{4}$ [compare the coefficient of $\beta_{1} \beta_{0}$ in Eq. (47) with $r_{3}^{(2)}$ in Eq. (37)]. Thus, strictly based on the algebraic decomposition of the coefficients at previous orders there is no way to deter- 
mine the coefficient of $\beta_{1} \beta_{0}$ at order $\bar{a}\left(Q^{2}\right)^{5}$. Additional information, namely the values of $\phi_{1}^{(1,1)}, \phi_{1}^{(2,0)}$ and $\phi_{1}^{(0,2)}$, is required. In the Abelian case, where the diagrammatic identification of the skeleton structure is transparent, it should be straightforward to calculate these moments separately. In non-Abelian theory this is not yet achievable.

The need to identify the skeleton structure, as a preliminary stage to writing the decomposition of the coefficients (and thus also to BLM scale setting), may actually arise at lower orders if several skeletons appear at the same order. As mentioned in the previous section, even in the Abelian case the assumed form of the skeleton expansion (2) is oversimplified in this sense and should be generalized to include several different $s_{i} R_{i}\left(Q^{2}\right)$ terms at any order $i$. In the nonAbelian case one should expect the set of skeleton diagrams to be larger.

The simplest possibility to imagine is that the momentum distribution functions $\phi_{i}$ are $N_{c}$ independent. It is then natural to expect that at any given order there will be several skeletons, where each of them is characterized by its own color group structure. For example, let us assume that in the case of the QCD correction to the photon vacuum polarization $s_{1} R_{1}\left(Q^{2}\right)$ should be replaced by a sum of three skeleton terms, $s_{1}^{p} R_{1}^{p}\left(Q^{2}\right)+s_{1}^{n p} R_{1}^{n p}\left(Q^{2}\right)+s_{1}^{3 g} R_{1}^{3 g}\left(Q^{2}\right)$, where the first two terms correspond to double gluon exchange (which exist in Abelian theory) - the planar ( $p)$ and the non-planar $(n p)$ skeleton diagrams - and the last term corresponds to the three gluon vertex skeleton diagram ${ }^{7}$ (which vanishes in the Abelian limit). Each of these three terms contributes starting at order $\bar{a}^{2}$. In this case, the skeleton decomposition of Eq. (45) appears to be too naive: each of these skeleton terms has its own momentum flow. In particular, if the BLM series is to have a one-to-one correspondence with the skeleton expansion, one should write

$$
\begin{aligned}
a_{R}\left(Q^{2}\right)= & \bar{a}\left(k_{0}^{2}\right)+s_{1}^{p} \bar{a}\left(k_{1, p}^{2}\right)^{2}+s_{1}^{n p} \bar{a}\left(k_{1, n p}^{2}\right)^{2}+s_{1}^{3 g} \bar{a}\left(k_{1,3 g}^{2}\right)^{2} \\
& +\cdots .
\end{aligned}
$$

To arrive at such a BLM series one should further decompose the coefficients in Eq. (45) as follows:

$$
\begin{aligned}
a_{R}= & \bar{a}+\left[s_{1}+r_{1}^{(1)} \beta_{0}\right] \bar{a}^{2}+\left[s_{2}+\left(s_{1}^{p} r_{2, p}^{(1)}+s_{1}^{n p} r_{2, n p}^{(1)}\right.\right. \\
& \left.\left.+s_{1}^{3 g} r_{2,3 g}^{(1)}\right) \beta_{0}+r_{2}^{(2)} \beta_{0}^{2}+r_{1}^{(1)} \beta_{1}\right] \bar{a}^{3}+\cdots
\end{aligned}
$$

where

$$
s_{1}^{p} \equiv \sigma_{1}^{p} C_{F}
$$

\footnotetext{
${ }^{7}$ Note that the three gluon vertex, which is a fundamental vertex in the theory, cannot be considered as just renormalizing the gluon propagator and the quark vertex. Part of it must define a new skeleton. This is in contrast to other diagrams appearing at this order which just renormalize the propagators or the quark vertex, and are therefore not candidates for new skeleton structures.
}

$$
\begin{aligned}
& s_{1}^{n p} \equiv \sigma_{1}^{n p}\left(C_{F}-\frac{1}{2} C_{A}\right) \\
& s_{1}^{3 g} \equiv\left(\sigma_{A}+\frac{1}{2} \sigma_{1}^{n p}\right) C_{A}
\end{aligned}
$$

and

$$
\begin{aligned}
s_{1}= & \left(\sigma_{1}^{p}+\sigma_{1}^{n p}\right) C_{F}+\sigma_{1}^{3 g} C_{A}=\sigma_{1}^{p} C_{F}+\sigma_{1}^{n p}\left(C_{F}-\frac{1}{2} C_{A}\right) \\
& +\left(\sigma_{A}+\frac{1}{2} \sigma_{1}^{n p}\right) C_{A} .
\end{aligned}
$$

Here the combination $\left(C_{F}-\frac{1}{2} C_{A}\right)$ corresponding to the nonplanar skeleton $(n p)$ is suppressed in the large $N_{c}$ limit. $^{8}$ Since the two Abelian parts of $s_{1}$, namely $s_{1}^{p}$ and $s_{1}^{n p}$, are separately calculable, the coefficients $r_{2, p}^{(1)}, r_{2, n p}^{(1)}$ and $r_{2,3 g}^{(1)}$ are uniquely determined from $r_{2}$. Thus, in this example the color group structure plus the Abelian skeleton decomposition allow one to determine the non-Abelian skeleton decomposition. In the general case, where more skeleton structures are possible, this information will not suffice, and the decomposition of the coefficients will require a more complete understanding of the non-Abelian skeleton expansion.

To summarize, we have seen that by tracing the flavor dependence of the perturbative coefficients in the skeleton scheme, one can identify the contribution of the different skeleton terms. This procedure allows us to "reconstruct" the skeleton expansion algebraically from the calculated coefficients as summarized by Eq. (45). This decomposition implies a unique BLM scale setting which has a one-to-one correspondence with the skeleton expansion. We also learned that there are several limitations to the algebraic procedure which can probably be resolved only by explicit diagrammatic identification of the skeleton structures and the skeleton effective charge. These limitations include the need to

(a) treat separately contributions from skeleton structures which involve fermion loops (in the Abelian case these are just the light-by-light type diagrams),

(b) identify separately the different moments $\phi_{i}^{(j, k)}$ of a given momentum distribution function which appear as a sum (with any $j$ and $k$ such that $j+k=n$ ) in the perturbative coefficients of $\beta_{0}^{n} \bar{a}^{1+i+n}$, and

(c) identify separately the contributions of different skeleton terms which happen to appear at the same order in $\bar{a}$.

\section{USING THE ECH METHOD IN THE FRAMEWORK OF THE SKELETON EXPANSION}

As we saw in the previous section, the essential ingredient of the BLM approach, which crucially relies on the skeleton expansion, is to disentangle running-coupling effects and

\footnotetext{
${ }^{8} \mathrm{In} \mathrm{SU}\left(N_{c}\right)$ the combination $\left(C_{F}-\frac{1}{2} C_{A}\right)$ is sub-leading in $N_{c}$ compared to $C_{A}=N_{c}$ and $C_{F}=\left(N_{c}^{2}-1\right) /\left(2 N_{c}\right)$.
} 
treat them separately from the remaining expansion. Technically, this is realized by performing a skeleton decomposition. Running coupling effects can then be resummed in various ways, aiming at the approximation of the skeleton integrals $R_{i}$. First, there is the possibility to perform a full all-order resummation by evaluating the integrals using some regularization in the infrared region (see e.g. [31]). This, however, requires the computation of the corresponding momentum distribution function, which can currently be done only at the level of the leading skeleton term. At the more modest level, the skeleton decomposition itself, Eq. (45), provides some information about the first few moments of the momentum distribution functions which is then used (Sec. III) to perform BLM scale setting. Alternatively, the same information can be used to approximate the skeleton terms $R_{i}\left(Q^{2}\right)$ in the ECH method, which is particularly fit to deal with running coupling effects [5] (see also [42]). We shall see that this method has close relations with the scale setting procedure, but it also has some advantages over the latter.

In this section we demonstrate how the ECH method can be used to provide resummation of running coupling effects in the framework of the skeleton expansion. The basic idea is that each skeleton term $R_{i}\left(Q^{2}\right)$ in our ansatz (2) is a renormalization-group invariant effective charge raised to some power; i.e., one writes $R_{i}\left(Q^{2}\right) \equiv\left[a_{R_{i}}\left(Q^{2}\right)\right]^{i+1}$ instead of Eq. (19). Thus $a_{R_{i}}\left(Q^{2}\right) \equiv\left[R_{i}\left(Q^{2}\right)\right]^{1 /(i+1)}$ can be simply evaluated in the ECH method [5], avoiding any explicit scale-setting procedure. In this method $a_{R_{i}}$ is computed by inverting the integrated renormalization-group equation,

$$
\ln Q^{2} / \Lambda_{R_{i}}^{2}=\int_{0}^{a_{R_{i}}} \frac{d a}{\beta_{R_{i}}(a)} .
$$

Finally, the observable $a_{R}\left(Q^{2}\right)$ will be written as [cf. Eqs. (2) and (18)]

$$
a_{R}\left(Q^{2}\right)=a_{R_{0}}\left(Q^{2}\right)+s_{1} a_{R_{1}}\left(Q^{2}\right)^{2}+s_{2} a_{R_{2}}\left(Q^{2}\right)^{3}+\cdots .
$$

Consider first the effective charge defined by the leading skeleton term $a_{R_{0}} \equiv R_{0}$ as expanded in Eq. (8). From the next-to-leading order coefficient in this equation it follows that the ratio between the two scale parameters characterizing $a_{R_{0}}$ and $\bar{a}$ is

$$
\Lambda_{R_{0}}^{2} / \bar{\Lambda}^{2}=e^{-r_{1}^{(1)}} .
$$

This ratio is fully determined by the center of the momentum distribution function pwhich is also the leading order BLM scale shift $t_{0,0}$; cf. Eq. (14)] and is not modified at higher orders. The latter affect just the corresponding ECH $\beta$ function, $\beta_{R_{0}}\left(a_{R_{0}}\right) \equiv d a_{R_{0}} / \ln Q^{2}$. Using the next-to-next-toleading order expansion of $a_{R_{0}}$ in terms of $\bar{a}$ and applying the general relation between effective charges [5], we have

$$
\beta_{2}^{R_{0}}=\bar{\beta}_{2}+\beta_{0}\left(r_{2}-r_{1}^{2}\right)-\beta_{1} r_{1},
$$

where $\bar{\beta}_{2}$ and $\beta_{2}^{R_{0}}$ are the three-loop $\beta$ function coefficients of the skeleton coupling and of $a_{R_{0}}$, respectively. Using now $r_{1}$ and $r_{2}$ of Eq. (8) we obtain

$$
\beta_{2}^{R_{0}}=\bar{\beta}_{2}+\left[r_{2}^{(2)}-\left(r_{1}^{(1)}\right)^{2}\right] \beta_{0}^{3}=\bar{\beta}_{2}+M_{2} \beta_{0}^{3} .
$$

This means that for any momentum distribution $\phi_{0}, \beta_{2}^{R_{0}}$ is simply a sum of a universal piece $\bar{\beta}_{2}$, which characterizes the skeleton coupling, and an observable-dependent piece, namely the width of $\phi_{0}$ (see Sec. III) multiplied by $\beta_{0}^{3}$.

Recall that the three-loop $\beta$ function coefficient in the skeleton scheme $\bar{\beta}_{2}$ is a polynomial of order 2 in $\beta_{0}$, namely $\bar{\beta}_{2}=\bar{\beta}_{2,0}+\bar{\beta}_{2,1} \beta_{0}+\bar{\beta}_{2,2} \beta_{0}^{2}$ [see footnote 1 following Eq. (9)]. Therefore $\beta_{2}^{R_{0}}$ is given by

$$
\beta_{2}^{R_{0}}=\bar{\beta}_{2,0}+\bar{\beta}_{2,1} \beta_{0}+\bar{\beta}_{2,2} \beta_{0}^{2}+\left[r_{2}^{(2)}-\left(r_{1}^{(1)}\right)^{2}\right] \beta_{0}^{3} .
$$

In the large $\beta_{0}$ limit $\beta_{2}^{R_{0}}$ is dominated by the last term, namely by the width of momentum distribution $\phi_{0}$. In this case it is therefore the width which controls the convergence of the ECH $\beta$ function, i.e. the accuracy of the calculated effective charge. Note that the same parameter controls the accuracy of the leading order BLM approximation $[14,17]$. Away from the large $\beta_{0}$ limit, a small width implies proximity of $\beta_{2}^{R_{0}}$ and $\bar{\beta}_{2}$ (see the Appendix). Thus only if the universal $\bar{\beta}_{2}$ is not large does a small width imply smallness of $\beta_{2}^{R_{0}}$, i.e. good convergence of the effective charge approach applied to $R_{0}$. Similarly at the four-loop level, one gets

$$
\beta_{3}^{R_{0}}=\bar{\beta}_{3}+2 M_{3} \beta_{0}^{4}+5 M_{2} \beta_{1} \beta_{0}^{3} .
$$

As usual [5] the effective charge $a_{R_{0}}$ is characterized by the scale ratio $\Lambda_{R_{0}}^{2} / \bar{\Lambda}^{2}$ and $\beta$ function coefficients. The same holds for higher skeleton terms. For example, it follows from Eq. (36) that $a_{R_{1}} \equiv\left(R_{1}\right)^{1 / 2}$ is characterized by $\Lambda_{R_{1}}^{2} / \bar{\Lambda}^{2}$ $=e^{-r_{2}^{(1) / 2}}$ and $\beta_{2}^{R_{1}}=\bar{\beta}_{2}+\frac{1}{2}\left[r_{3}^{(2)}-\frac{3}{4}\left(r_{2}^{(1)}\right)^{2}\right] \beta_{0}^{3}$. Note that these are the same combinations appearing in the BLM scale shift for $R_{1}$, Eq. (42).

It is also worth noting that the suggested effective charge approach yields a result identical to the BLM scale setting method applied in the skeleton scheme, in the approximation where the $\beta$ functions of the effective charges associated with the various skeletons $\beta^{R_{j}}$ are all replaced by the skeleton coupling $\beta$ function, $\bar{\beta}$. This is equivalent to assuming that, except the average, all the central moments of the momentum distribution functions vanish identically. Then both approaches effectively yield a multi-scale series where the scales correspond to the average momentum flowing in each skeleton diagram, $k_{i, 0}$.

To conclude, we have shown that the explicit scale-setting procedure can be replaced by the ECH method. One advantage is that the latter does not suffer from the scheme and scale ambiguities still present [see footnote 5 before Eq. (20)] in the series for the BLM scales. We stress that our 
example here heavily relies on the specific ansatz assumed for the skeleton expansion. However, contrary to the BLM scale setting method, the suggested effective charge approach would apply equally well to more general cases where e.g. the two couplings in Eq. (4) are different.

\section{BLM AND CONFORMAL RELATIONS}

Let us now consider the general BLM scale-setting method, where the scheme is not necessarily the one of the skeleton effective charge, and no correspondence with the skeleton expansion is sought for. Then any scale-setting procedure which yields an expansion of the form (18) with $N_{f}$ independent $c_{i}$ coefficients and scale shifts which are power series in the coupling (23) is legitimate. We saw that under these requirements there is no unique procedure for setting the BLM scale beyond the leading order $\left(k_{0,0}\right)$. Nevertheless, as we now show, the coefficients $c_{i}$ are uniquely defined. In fact, the $c_{i}$ have a precise physical interpretation as the "conformal coefficients" relating $a_{R}$ and $a$ in a conformal theory defined by

$$
\beta(a)=-\beta_{0} a^{2}-\beta_{1} a^{3}+\cdots=0 .
$$

To go from real-world QCD to a situation where such a conformal theory exists one has to tune $N_{f}$ : when $N_{f}$ is set large enough (but still below $\frac{11}{2} N_{c}$, the point where asymptotic freedom is lost) $\beta_{1}$ is negative while $\beta_{0}$ is positive and small. Then the perturbative $\beta$ function has a zero at $a_{\mathrm{FP}} \simeq-\beta_{0} / \beta_{1}$; i.e., there is a non-trivial infrared fixed point [34-38]. The perturbative analysis is justified if $\beta_{0}$, and hence $a_{\mathrm{FP}}$ is small enough.

Physically, the existence of an infrared fixed point in QCD means that correlation functions are scale invariant at large distances. This contradicts confinement which requires a characteristic distance scale. In particular, when $\beta_{0} \rightarrow 0$ the infrared coupling is vanishingly small. Then it is quite clear that a non-perturbative phenomenon such as confinement will not persist. The phase of the theory where the infrared physics is controlled by a fixed point is called the conformal window. In this work we are not concerned with the physics in the conformal window. ${ }^{9}$ We shall just use formal expansions which have a particular meaning in this phase.

The BLM coefficients $c_{i}$ are by definition $N_{f}$ independent. Therefore the expansion of $a_{R}$ according to Eq. (18) is valid, with the same $c_{i}$ 's both in the real world QCD and in the conformal window. In the conformal window a generic coupling $a\left(k^{2}\right)$ flows in the infrared to a well-defined limit $a\left(k^{2}=0\right) \equiv a_{\mathrm{FP}}$. In particular, Eq. (18) becomes

$$
a_{R}^{\mathrm{FP}}=a_{\mathrm{FP}}+c_{1} a_{\mathrm{FP}}^{2}+c_{2} a_{\mathrm{FP}}^{3}+c_{3} a_{\mathrm{FP}}^{4}+\cdots
$$

where we used the fact that the $k_{i}$ 's are proportional to $Q$, which follows from their definition $k_{i}^{2}=Q^{2} \exp \left(-t_{i}\right)$, together with the observation that the scale shifts $t_{i}$ in Eq. (23)

\footnotetext{
${ }^{9}$ In [38] this phase is investigated from the point of view of perturbation theory in both QCD and supersymmetric QCD.
}

at any finite order are just constants when $a\left(Q^{2}\right) \rightarrow a_{\mathrm{FP}}$. Equation (60) is simply the perturbative relation between the fixed-point values of the two couplings (or effective charges) $a_{R}$ and $a$.

Note that in this discussion we ignored the complication discussed at the end of Sec. III, concerning the possibility of applying BLM scale setting in the case of several skeletons contributing at the same order [cf. Eq. (48)]. In this case the argument above holds as well, while the conformal coefficients will be the sum of all BLM coefficients appearing at the corresponding order. For the example considered in Sec. III, Eq. (49), we would then have $c_{1}=s_{1}=s_{1}^{p}+s_{1}^{n p}+s_{1}^{3 g}$.

According to the general argument above, the BLM coefficients Eq. (18) should coincide with the conformal coefficients in (60). In the next section we calculate conformal coefficients directly and check this statement explicitly in the first few orders.

\section{CALCULATING CONFORMAL COEFFICIENTS}

Let us now investigate the relation between the conformal coefficients $c_{i}$ appearing in Eq. (60) and the perturbative coefficients $r_{i}$.

For this purpose, it is useful to recall the Banks-Zaks expansion: solving the equation $\beta(a)=0$ in Eq. (59) for such $N_{f}$ where $\beta_{0}$ is small and positive and $\beta_{1}$ is negative, we obtain $a_{\mathrm{FP}} \simeq-\beta_{0} / \beta_{1}>0$. If we now tune $N_{f}$ towards the limit $\frac{11}{2} N_{c}$ from below, $\beta_{0}$ and therefore $a_{\mathrm{FP}}$ become vanishingly small, which justifies the perturbative analysis [26,27]. In particular, it justifies neglecting higher orders in the $\beta$ function as a first approximation. In order to take into account the higher orders in the $\beta$ function, one can construct a power expansion solution of the equation $\beta(a)=0$, with the expansion parameter as the leading order solution:

$$
a_{0} \equiv-\frac{\beta_{0}}{\left.\beta_{1}\right|_{\beta_{0}=0}}=\frac{\beta_{0}}{-\beta_{1,0}} .
$$

In the last equality we defined $\beta_{1} \equiv \beta_{1,0}+\beta_{1,1} \beta_{0}$ where $\beta_{i, j}$ are $N_{f}$ independent. Similarly, we define, ${ }^{10}$ for later use,

$$
\beta_{2} \equiv \beta_{2,0}+\beta_{2,1} \beta_{0}+\beta_{2,2} \beta_{0}^{2}+\beta_{2,3} \beta_{0}^{3} .
$$

We shall assume that the coupling $a$ has the following Banks-Zaks expansion:

$$
a_{\mathrm{FP}}=a_{0}+v_{1} a_{0}^{2}+v_{2} a_{0}^{3}+v_{3} a_{0}^{4}+\cdots
$$

where $v_{i}$ depend on the coefficients of $\beta(a)$; see e.g. [37]. For instance, the first Banks-Zaks coefficient is

$$
v_{1}=\beta_{1,1}-\frac{\beta_{2,0}}{\beta_{1,0}} .
$$

Suppose that the perturbative expansion of $a_{R}\left(Q^{2}\right)$ in terms of $a\left(Q^{2}\right)$ is given by

\footnotetext{
${ }^{10} \mathrm{We}$ recall that in the skeleton scheme $\bar{\beta}_{2,3}=0$.
} 


$$
a_{R}\left(Q^{2}\right)=a\left(Q^{2}\right)+r_{1} a\left(Q^{2}\right)^{2}+r_{2} a\left(Q^{2}\right)^{3}+\cdots .
$$

Based on the fact that $r_{i}$ are polynomials of order $i$ in $N_{f}$, and that $a_{0}$ is linear in $N_{f}$, one can uniquely write a decomposition of $r_{i}$ into polynomials in $a_{0}$ with $N_{f}$-independent coefficients:

$$
\begin{aligned}
& r_{1}=r_{1,0}+r_{1,1} a_{0} \\
& r_{2}=r_{2,0}+r_{2,1} a_{0}+r_{2,2} a_{0}{ }^{2} \\
& r_{3}=r_{3,0}+r_{3,1} a_{0}+r_{3,2} a_{0}{ }^{2}+r_{3,3} a_{0}{ }^{3}
\end{aligned}
$$

and so on. For convenience we expand here in $a_{0}$ rather than in $\beta_{0}$. The relations with the "skeleton decomposition" of $r_{1}$ and $r_{2}$ in Eqs. (16) and (21) [or in Eq. (45)] are the following:

$$
\begin{array}{ll}
r_{1,0}=r_{1}^{(0)} & r_{2,0}=r_{2}^{(0)}+\beta_{1,0} r_{1}^{(1)} \\
r_{1,1}=-\beta_{1,0} r_{1}^{(1)} & r_{2,1}=-\beta_{1,0} r_{2}^{(1)} r_{1}^{(0)}-\beta_{1,0} \beta_{1,1} r_{1}^{(1)} \\
r_{2,2}=\beta_{1,0}^{2} r_{2}^{(2)} . &
\end{array}
$$

For $r_{3}$ we have, based on Eq. (45),

$$
r_{3,0}=r_{3}^{(0)}+r_{2}^{(1)} r_{1}^{(0)} \beta_{1,0}+r_{1}^{(1)} \beta_{2,0} .
$$

Using Eq. (65) at $Q^{2}=0$ with Eq. (66) and the BanksZaks expansion for $a_{\mathrm{FP}}$, Eq. (63), it is straightforward to obtain the Banks-Zaks expansion for $a_{R}^{\mathrm{FP}}$ :

$$
a_{R}^{\mathrm{FP}}=a_{0}+w_{1} a_{0}^{2}+w_{2} a_{0}^{3}+w_{3} a_{0}^{4}+\cdots
$$

with

$$
\begin{aligned}
& w_{1}=v_{1}+r_{1,0} \\
& w_{2}=v_{2}+2 r_{1,0} v_{1}+r_{1,1}+r_{2,0} \\
& w_{3}=v_{3}+2 r_{1,0} v_{2}+r_{1,0} v_{1}^{2}+2 r_{1,1} v_{1}+3 r_{2,0} v_{1}+r_{2,1}+r_{3,0} .
\end{aligned}
$$

Having the two Banks-Zaks expansions, one can also construct the series which relates two effective charges $a_{R}^{\mathrm{FP}}$ and $a_{\mathrm{FP}}$ at the fixed-point. Inverting the series in Eq. (63) one obtains $a_{0}$ as a power series in $a_{\mathrm{FP}}$,

$$
a_{0}=a_{\mathrm{FP}}+u_{1} a_{\mathrm{FP}}^{2}+u_{2} a_{\mathrm{FP}}^{3}+u_{3} a_{\mathrm{FP}}^{4}+\cdots
$$

with $u_{1}=-v_{1}$ and $u_{2}=v_{1}^{2}-v_{2}$ etc. Substituting Eq. (71) into Eq. (69) one obtains the "conformal expansion" of $a_{R}^{\mathrm{FP}}$ in terms of $a_{\mathrm{FP}}$ according to Eq. (60) with

$$
\begin{aligned}
& c_{1}=r_{1,0} \\
& c_{2}=r_{1,1}+r_{2,0} \\
& c_{3}=-r_{1,1} v_{1}+r_{2,1}+r_{3,0}
\end{aligned}
$$

$$
c_{4}=2 r_{1,1} v_{1}^{2}-r_{1,1} v_{2}-r_{2,1} v_{1}+r_{2,2}+r_{3,1}+r_{4,0} .
$$

Thus the coefficients $v_{i}$ of the Banks-Zaks expansion (63) and the coefficients $r_{i}$ of Eq. (65) are sufficient to determine the conformal coefficients $c_{i}$ to any given order.

Clearly, the Banks-Zaks expansions (63) and (69) and the conformal expansion of one fixed point in terms of another, Eq. (60), are closely related. Strictly speaking, both types of expansions are meaningful only in the conformal window. However, we saw that the coefficients of Eq. (60) coincide with the ones of the BLM series (18) which is useful in real world QCD. We recall that the general argument in the previous section does not depend on the specific BLM scalesetting prescription used, provided that the scales $k_{i}$ are proportional to $Q$ and the $c_{i}$ 's are $N_{f}$ independent. Comparing explicitly $c_{1}, c_{2}$ and $c_{3}$ in Eq. (72) with the BLM coefficients obtained in the previous section, namely $c_{i}=r_{i}^{(0)}$, we indeed find that they are equal [compare using Eqs. (67), (68) and (64)]. In particular, the "skeleton decomposition" of Eq. (45), which can be formally performed in any scheme, provides an alternative way to compute conformal coefficients.

\section{EXAMPLES}

The skeleton expansion assumption implies that the skeleton (conformal) coefficients $s_{i}$ are free of running coupling effects. In particular, contrary to the standard perturbative coefficients in a standard scheme such as $\overline{\mathrm{MS}}$, the large order behavior of conformal coefficients is not dictated by renormalon factorial increase, and should therefore be softer.

In other words, the effective convergence of the fixedpoint relation (60) where $a$ is taken as the skeleton coupling effective charge $\bar{a}$ is expected to be better than standard perturbative expansions. As we shall see in Sec. VII C, this expectation is not restricted to the skeleton scheme but applies also to general conformal relations, e.g. between two physical effective charges. In addition, if we assume that the skeleton coupling $\beta$ function itself is renormalon free, it follows that also the Banks-Zaks expansion of a generic physical quantity $a_{R}$ is renormalon free. This is because the latter assumption implies that the Banks-Zaks expansion of $\bar{a}$, Eq. (63), is free of renormalons, and then, by substituting it in the renormalon-free conformal relation between the observable $a_{R}$ and $\bar{a}$, one recovers the Banks-Zaks expansion of $a_{R}$, which must therefore be renormalon free as well.

Thus, the general expectation is that all conformal and Banks-Zaks relations are free of renormalons and have better convergence properties. Our purpose here is to examine through available examples in QCD whether this expectation is realized. Indeed, as we recall below, it has been noted by several authors (e.g. in $[12,33,18,36,37]$ ) that conformal coefficients and Banks-Zaks coefficients are typically small. We would like to interpret these observations based on the assumed skeleton expansion and relate them to the absence of renormalons. As concrete examples we shall concentrate on the following observables: 
(a) The Adler $D$ function,

$$
D\left(Q^{2}\right)=Q^{2} \frac{d \Pi\left(Q^{2}\right)}{d Q^{2}} \equiv N_{c} \sum_{f} e_{f}^{2}\left[1+\frac{3}{4} C_{F} a_{D}\right]
$$

where $a_{D}$ is normalized as an effective charge, and $\Pi\left(Q^{2}\right)$ is the electromagnetic vacuum polarization:

$$
\begin{gathered}
4 \pi^{2} i \int d^{4} x e^{i q \cdot x}\left\langle 0\left|T\left\{j^{\mu}(x), j^{\nu}(0)\right\}\right| 0\right\rangle \\
=\left(q^{\mu} q^{\nu}-q^{2} g^{\mu \nu}\right) \Pi\left(Q^{2}\right) .
\end{gathered}
$$

(b) The polarized Bjorken sum rule for electron nucleon deep-inelastic scattering,

$$
\int_{0}^{1}\left[g_{1}^{p}\left(x, Q^{2}\right)-g_{1}^{n}\left(x, Q^{2}\right)\right] d x \equiv \frac{g_{A}}{6}\left[1-\frac{3}{4} C_{F} a_{g_{1}}\right] .
$$

(c) The non-polarized Bjorken sum rule for neutrino nucleon deep-inelastic scattering,

$$
\int_{0}^{1} d x\left[F_{1}^{\bar{\nu} p}\left(x, Q^{2}\right)-F_{1}^{\nu n}\left(x, Q^{2}\right)\right] \equiv 1-\frac{C_{F}}{2} a_{F_{1}} .
$$

(d) The static potential,

$$
V\left(Q^{2}\right) \equiv-4 \pi^{2} C_{F} \frac{a_{V}}{Q^{2}}
$$

In all four cases perturbative calculations have been performed (Refs. [43-46], respectively) up to the next-to-nextto-leading order $r_{2}$ in Eq. (1).

For later comparison with conformal relations, we quote some numerical values of the coefficients in the standard perturbative expansion in $a_{\overline{\mathrm{MS}}} \equiv a_{\overline{\mathrm{MS}}}\left(Q^{2}\right)$ for the vacuum polarization $D$ function (73),

$$
\begin{array}{rllll}
a_{D}=a_{\overline{\mathrm{MS}}}+d_{1} & a_{\overline{\mathrm{MS}}}^{2}+d_{2} & a_{\overline{\mathrm{MS}}}^{3}+\cdots & \\
2.0 & & 18.2 & \\
1.6 & & 6.4 & N_{f}=0 \\
0.14 & & -27.1 & N_{f}=3 \\
1.06 & & 14.0 & N_{f}=16 \\
& & & N_{f}=0-16
\end{array}
$$

and for the polarized Bjorken sum rule (75),

$$
\begin{aligned}
& a_{g_{1}}=a \overline{\mathrm{MS}}+k_{1} \quad a_{\overline{\mathrm{MS}}}^{2}+k_{2} \quad a_{\overline{\mathrm{MS}}}^{3}+\cdots \\
& 4.6 \\
& 3.5 \\
& -0.75 \\
& 2.1 \\
& 41.4 \\
& N_{f}=0 \\
& 20.2 \\
& N_{f}=3 \\
& -34.8 \\
& N_{f}=16 \\
& 21.0 \\
& N_{f}=0-16
\end{aligned}
$$

where in the first three lines in Eqs. (78) and (79) the coefficients are evaluated at given $N_{f}$ values, while the last line corresponds to an average of $\left|r_{i}\right|$ in the range $N_{f}=0-16$.

We see that the coefficients in a running coupling expansion in the $\overline{\mathrm{MS}}$ scheme increase fast already at the available next-to-next-to-leading order. This increase has been discussed in connection with renormalons, for example in [4]. A priori, it is hard to expect that the large-order behavior of the series will show up already in the first few leading orders. We mention, however, that in Ref. [47] the Bjorken sum rule series (for $N_{f}=3$ ) was analyzed in the Borel plane based on the three known coefficients, indicating that the first infrared renormalon at $p=1$ does show up.

\section{A. Banks-Zaks expansion}

Let us now compare the magnitude of the coefficients in the standard expansion, e.g. in Eqs. (78) and (79), to that of conformal coefficients. For the latter, one can choose to examine conformal relations between effective charges (see Sec. VII C) or the Banks-Zaks expansion.

The Banks-Zaks expansion for the fixed-point value of the vacuum polarization $D$ function (73) is

$$
a_{\mathrm{D}}^{\mathrm{FP}}=a_{0}+1.22 a_{0}{ }^{2}+0.23 a_{0}{ }^{3}+\cdots
$$

whereas for the Bjorken sum rule it is

$$
a_{g_{1}}^{\mathrm{FP}}=a_{0}+0.22 a_{0}{ }^{2}-1.21 a_{0}{ }^{3}+\cdots .
$$

Comparing Eqs. (81) and (80) with the corresponding running coupling expansions in the $\overline{\mathrm{MS}}$ scheme, namely Eqs. (78) and (79), the difference in magnitude of the coefficients is quite remarkable $[36,37]$. Taking into account the fact that the coefficient of $a_{0}^{i+1}$ contains, among other terms, a $C_{A}^{i}$ $=3^{i}$ term, this fast apparent convergence seems rather sur- 
prising. From this point of view, the absence of renormalons may not be considered a sufficient explanation.

For the non-polarized Bjorken sum rule defined by Eq. (82), the Banks-Zaks coefficients are even smaller,

$$
a_{\mathrm{F}_{1}}^{\mathrm{FP}}=a_{0}-0.45 a_{0}^{2}+0.16 a_{0}^{3}+\cdots,
$$

and exhibit an impressive cancelation of numerical terms appearing in the running coupling coefficients [37]. The static potential shows a different behavior. In this case the Banks-Zaks expansion $[37,38]$

$$
a_{\mathrm{V}}^{\mathrm{FP}}=a_{0}-0.86 a_{0}^{2}+10.99 a_{0}^{3}+\cdots
$$

has a significantly larger next-to-next-to-leading order coefficient. Taking into account the numerically large color group factor $C_{A}{ }^{2}=9$, the magnitude of this next-to-next-toleading order coefficient is quite reasonable.

Another physical quantity for which the Banks-Zaks coefficients are relatively large is the critical exponent $\hat{\gamma}$ $[27,36-38]$ :

$$
\hat{\gamma}=\left.\frac{1}{\beta_{0}} \frac{d \beta(a)}{d a}\right|_{a=a_{\mathrm{FP}}}
$$

where

$$
\hat{\gamma}=a_{0}+4.75 a_{0}{ }^{2}-8.89 a_{0}{ }^{3}+\cdots .
$$

Since this quantity does not depend on $Q^{2}$, there is no direct comparison between a running coupling expansion and the Banks-Zaks expansion.

To conclude, we have seen that the Banks-Zaks coefficients for physical quantities typically have smaller coefficients compared to the standard running coupling expansion. In some cases, their convergence is surprisingly good, even taking into account the absence of running-coupling effects.

\section{B. Conformal relations in the skeleton scheme}

Examining the Banks-Zaks expansion we found that the coefficients are significantly smaller than standard runningcoupling coefficients. The same conclusion would follow from examining direct conformal relations between observables. The coefficients of such relations (see Sec. VIIC) are not only small, but also exhibit a remarkable simplicity [12]. Both the smallness and the simplicity of these coefficients seem a natural consequence of the conformal limit. The smallness, in particular, is naturally attributed to the absence of running-coupling effects.

The first step in trying to substantiate this statement in the framework of the postulated skeleton expansion is to consider the conformal relations in the skeleton scheme. It is natural to expect that conformal relations between observables and the skeleton coupling will be small, thus explaining the above observations.

To this end, let us consider now the conformal relation in the skeleton scheme (60) as defined by the pinch technique. Since the skeleton coupling $\bar{a}$ has been identified only at the one-loop level (12), our information on the coefficients $s_{i}$ is quite limited: by a direct calculation [using the next-toleading order coefficient $r_{1}$ and either Eq. (16) or (72)] we can only determine $s_{1}$. For example, for the observables defined above it is

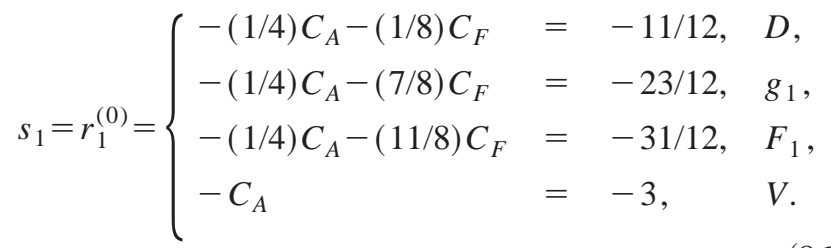

Note the absence of a $C_{F}$ term in the case of the static potential. This can be understood based on the Abelian limit, where it is known that this effective charge coincides with the skeleton coupling (there, the Gell-Mann-Low effective charge) up to light-by-light type corrections. Therefore the momentum distribution function of the leading skeleton term $\phi_{0}$ is just a $\delta$ function, $\phi_{0}\left(k^{2}\right)=\delta\left(k^{2}\right)$, and in the Abelian limit there are strictly no $\left(N_{f}\right.$-independent) sub-leading skeleton terms.

The higher-order coefficients $s_{i}$, for $i \geqslant 2$, depend on yet unknown characteristics of the skeleton coupling scheme. In particular, as we discuss in the Appendix, $s_{2}$ depends on the skeleton $\beta$ function coefficient $\bar{\beta}_{2}$. However, as can be seen in Eq. (A6), there, the dependence on this coefficient cancels in the difference of $s_{2}$ between any two observables, which is therefore calculable.

Without a diagrammatic identification of the skeleton structure, one cannot isolate skeletons with fermion loops attached to three gluons, which may appear at the order considered. Therefore we shall just treat the entire $N_{f}$ dependence (excluding Abelian light-by-light diagrams) as if it appears due to the running coupling, according to Eq. (45) where $s_{2}$ is $N_{f}$ independent. For the observables considered above we then find

$$
\begin{aligned}
s_{2}^{g_{1}}-s_{2}^{D}= & \frac{3}{8} C_{F} C_{A}+\frac{3}{4} C_{F}{ }^{2}=2.833 \\
s_{2}^{F_{1}}-s_{2}^{D}= & {\left[\frac{43}{12}+\frac{85}{6} \zeta_{3}-\frac{115}{6} \zeta_{5}\right] C_{A}{ }^{2} } \\
& +\left[-34 \zeta_{3}-\frac{75}{8}+\frac{95}{2} \zeta_{5}\right] C_{F} C_{A} \\
& +\left[\frac{21}{2}+\frac{47}{2} \zeta_{3}-35 \zeta_{5}\right] C_{F}{ }^{2} \\
= & 7.045 \\
s_{2}^{V}-s_{2}^{D}= & {\left[\frac{1}{4} \pi^{2}+\frac{43}{24}-\frac{1}{64} \pi^{4}\right] C_{A}{ }^{2}-\frac{25}{16} C_{F} C_{A}+\frac{23}{32} C_{F}^{2} } \\
= & 19.66 .
\end{aligned}
$$

This gives some estimate of the size of $s_{2}$ for these observables. The $s_{2}$ coefficients turn out to be larger than the 
Banks-Zaks coefficients quoted above (as well as the conformal coefficients in the relation between observables). They can even be comparable in size to the next-to-next-to-leading order coefficients in the $\overline{\mathrm{MS}}$ scheme. Thus the assumed form of the skeleton expansion does not provide a satisfactory explanation for the observed smallness of conformal coefficients. We stress again that we mistreated here the $N_{f}$ dependence which is associated with the skeleton structure, namely fermion loops attached to three gluons. Eventually, this will have some impact on the magnitude of the ( $N_{f}$ dependent) skeleton coefficients $s_{2}$, which we cannot evaluate at present.

\section{Direct relations between observables}

As we saw above knowledge about the skeleton coefficients is very limited beyond next-to-leading order. However, there is a way to consider systematically conformal relations avoiding the use of the skeleton scheme. Having renormalon-free conformal expansions (60) for two QCD observables in terms of the skeleton effective charge $\bar{a}$, one can eliminate the latter to obtain a direct conformal relation between the two observables. The existence of a skeleton expansion (2) for the two observables implies that this conformal relation is free of renormalons.

Conformal coefficients of this type can be computed either from the Banks-Zaks expansion (72) or in the framework of the BLM method, as the coefficients in a commensurate scale relation [12]. The latter can be obtained by applying the BLM method directly to the perturbative relation between two observable effective charges (and so it does not require identification of the skeleton coupling). However, it should be noted that whereas the one-to-one correspondence between the BLM series and the skeleton expansion specifies a unique scale-setting procedure when the skeleton scheme in used, the scale-setting procedure in direct relations between observables remains ambiguous. As explained in Secs. III and IV, the conformal coefficients themselves are uniquely determined, independently of the particular way the scales are set.

In addition to being numerically small, conformal coefficients in the direct relations between observables turn of to be simpler [12], in terms of color group factors and numerical $\zeta_{n}$ terms. This simplicity is naturally attributed to the conformal limit.

There is one example where a direct all-order conformal relation is known - this is the Crewther relation relating the vacuum polarization D-function effective charge $a_{D}$, defined by Eq. (73), with the polarized Bjorken sum-rule effective charge $a_{g_{1}}$, defined by Eq. (75). The Crewther relation is $[32,33,18]$

$$
a_{g_{1}}-a_{D}+\frac{3}{4} C_{F} a_{g_{1}} a_{D}=-\beta(a) T(a)
$$

where $T(a)$ is a power series in the coupling

$$
T(a)=T_{1}+T_{2} a+T_{3} a^{2}+\cdots
$$

and $T_{i}$ are polynomials in $N_{f}$.

If $a_{D}$ has a perturbative fixed point $a_{D}^{\mathrm{FP}}$, then it is convenient [37] to write the right-hand side (RHS) of Eq. (88) in terms of $a_{D}$. Here $\beta\left(a_{D}^{\mathrm{FP}}\right)=0$ and so the RHS vanishes at $a_{D}=a_{D}^{\mathrm{FP}}$ corresponding to the infrared limit. Therefore $a_{g_{1}}$ also freezes perturbatively, leading to the original conformal Crewther relation

$$
a_{g_{1}}^{\mathrm{FP}}=\frac{a_{D}^{\mathrm{FP}}}{1+\frac{3}{4} C_{F} a_{D}^{\mathrm{FP}}} .
$$

Taking $N_{c}=3$ we have $C_{F}=\frac{4}{3}$ and then the conformal coefficients are just one to any order in perturbation theory,

$$
a_{D}^{\mathrm{FP}}=a_{g_{1}}^{\mathrm{FP}}+\left(a_{g_{1}}^{\mathrm{FP}}\right)^{2}+\left(a_{g_{1}}^{\mathrm{FP}}\right)^{3}+\cdots .
$$

Being a geometrical series this conformal relation provides a nice example of a perturbative relation free of renormalon divergence. In addition, it exemplifies the simplicity of the conformal limit: here the conformal coefficients do not contain any non-Abelian $C_{A}$ terms.

As noted in [9] (see also [33]) it is possible to write for two generic observables $A$ and $B$, at two arbitrary scales $Q_{A}$ and $Q_{B}$, the following decomposition of the perturbative series relating the two:

$$
a_{A}=C_{A B}\left(a_{B}\right)+\beta\left(a_{B}\right) T_{A B}\left(a_{B}\right) .
$$

Here $C_{A B}$ is the "conformal part" of the series, i.e.

$$
C_{A B}\left(a_{B}\right)=a_{B}+c_{1} a_{B}^{2}+c_{2} a_{B}^{3}+\cdots
$$

where $c_{i}$ are the conformal coefficients appearing in the expansion of $a_{A}^{\mathrm{FP}}$ in terms of $a_{B}^{\mathrm{FP}}$, and $T_{A B}\left(a_{B}\right)$ is a perturbative series of the form (89). In other words the non-conformal part of the relation between the two observables is factorized [33] as $\beta\left(a_{B}\right) T_{A B}\left(a_{B}\right)$. Taking the limit $\beta \rightarrow 0$ then gives the conformal relation. In particular, one can write such a factorized relation between an observable effective charge and the skeleton coupling. Then the conformal coefficients $c_{i}$ in Eq. (93) are the skeleton coefficients $s_{i}$. Explicitly, this can be shown based on the skeleton decomposition of the series (45):

$$
\begin{aligned}
a_{R}= & {\left[\bar{a}+s_{1} \bar{a}^{2}+s_{2} \bar{a}^{3}+s_{3} \bar{a}^{4}+\cdots\right] } \\
& +\left[\beta_{0} \bar{a}^{2}+\beta_{1} \bar{a}^{3}+\bar{\beta}_{2} \bar{a}^{4}+\cdots\right] \\
& \times\left[r_{1}^{(1)}+\left(s_{1} r_{2}^{(1)}+r_{2}^{(2)} \beta_{0}\right) \bar{a}\right. \\
& \left.+\left(s_{2} r_{3}^{(1)}+s_{1} r_{3}^{(2)} \beta_{0}+r_{3}^{(3)} \beta_{0}^{2}+\frac{3}{2} r_{2}^{(2)} \beta_{1}\right) \bar{a}^{2}+\cdots\right] .
\end{aligned}
$$

Finally, we also quote the conformal relations between the vacuum polarization $D$ function and the non-polarized Bjorken sum rule (76), 


$$
a_{D}^{\mathrm{FP}}=a_{F_{1}}^{\mathrm{FP}}+1.67\left(a_{F_{1}}^{\mathrm{FP}}\right)^{2}+1.57\left(a_{F_{1}}^{\mathrm{FP}}\right)^{3}+\cdots,
$$

as well as the static potential (77),

$$
a_{D}^{\mathrm{FP}}=a_{V}^{\mathrm{FP}}+2.08\left(a_{V}^{\mathrm{FP}}\right)^{2}-7.16\left(a_{V}^{\mathrm{FP}}\right)^{3}+\cdots .
$$

Taking into account the $C_{A}{ }^{i}=3^{i}$ contribution to $c_{i}$, these expansions all seem well behaved.

\section{Expansions in the $\overline{\mathrm{MS}}$ scheme}

Finally, it is interesting to return to the expansion in the $\overline{\mathrm{MS}}$ scheme and examine the corresponding conformal relations. Such relations turn out to have large coefficients. For example,

$$
a_{D}^{\mathrm{FP}}=a \frac{\mathrm{FP}}{\mathrm{MS}}-0.083\left(a \frac{\mathrm{FP}}{\mathrm{MS}}\right)^{2}-23.22\left(a_{\overline{\mathrm{MS}}}^{\mathrm{FP}}\right)^{3}+\cdots
$$

and

$$
a_{g_{1}}^{\mathrm{FP}}=a_{\mathrm{MS}}^{\mathrm{FP}}-0.917\left(a_{\mathrm{MS}}^{\mathrm{FP}}\right)^{2}-22.39\left(a_{\mathrm{MS}}^{\mathrm{FP}}\right)^{3}+\cdots
$$

have large next-to-next-to-leading order coefficients, in striking contrast with the conformal relation (91) between $a_{D}^{\mathrm{FP}}$ and $a_{g_{1}}^{\mathrm{FP}}$. Note that these large conformal coefficients do not provide an explanation of the large coefficients in Eqs. (78) and (79). The former are by assumption independent of $N_{f}$, as opposed to the latter. For small $\beta_{0}$ (e.g. $\left.N_{f}=16\right)$ the negative sign (and eventually also the magnitude) of the full coefficient can presumably be attributed to the conformal part. However, for larger values of $\beta_{0}$, relevant to real world QCD, the non-conformal part clearly dominates making the full next-to-next-to-leading order coefficients positive.

These large conformal coefficients in Eqs. (97) and (98) are due to an intrinsic property of the $\overline{\mathrm{MS}}$ coupling, since they appear already at the level of the Banks-Zaks expansion $[37,38]$ :

$$
a_{\overline{\mathrm{MS}}}^{\mathrm{FP}}=a_{0}+1.1366 a_{0}{ }^{2}+23.2656 a_{0}{ }^{3}+\cdots .
$$

Note that $a_{\overline{\mathrm{MS}}}^{\mathrm{FP}}$ has, by far, a larger next-to-next-to-leading order Banks-Zaks coefficient compared to any known physical effective charge.

We stress that the large next-to-next-to-leading order coefficients in Eqs. (97), (98) and (99) are not associated with renormalons. The $\overline{\mathrm{MS}} \beta$ function, being defined through an ultraviolet regularization procedure, should not be sensitive to the infrared. Therefore infrared renormalons are not expected. It is more difficult to draw any firm conclusion concerning the absence of ultraviolet renormalons. Since there seems to be no reason to assume a skeleton structure or any other representation in the form of an integral over a running coupling, we suspect that ultraviolet renormalons do not exist there as well.

To conclude, the case of conformal relations in the $\overline{\mathrm{MS}}$ scheme teaches us not to associate automatically any large coefficient in QCD with running-coupling effects. Indeed, in field theory there are other sources of large coefficients, such as multiplicity of diagrams.

\section{CONCLUSIONS}

The fast growth of perturbative coefficients and the related renormalization scale and scheme ambiguities of perturbative expansions have greatly limited the predictive power of QCD. In many cases, this divergent behavior is predominantly due to running-coupling effects. The existence of an Abelian-like skeleton expansion in QCD would make it possible to disentangle in a unique way such effects, separating them from the conformal part of the perturbative expansion of a generic physical quantity. The effect of the running coupling could then be treated systematically to all orders in perturbation theory in a renormalization-scheme invariant manner by renormalon-type integrals. The normalization of these skeleton integrals is controlled by conformal coefficients that are hopefully better behaved, making the truncated skeleton expansion a better approximation to the physical observable compared to the standard perturbative expansion of the same order.

Resummation of running coupling effects has in many cases a significant role in phenomenology [4]. Direct resummation is currently restricted to the level of a single dressed gluon, where the Abelian large $N_{f}$ limit can be used. The formulation of perturbation theory in the form of a skeleton expansion has implications which go beyond the perturbative level. In particular, it provides a natural framework to deal together with the resummation and the related power corrections. The renormalon integral contains essential information on the type of power corrections one should expect for a given observable. Moreover, it can be used to combine [30,31] such power corrections with the perturbative expansion avoiding double counting or dependence on the particular prescription used to regularize infrared renormalons. These aspects were discussed in detail in [31] for the example of the average thrust.

In this paper we have concentrated on the conformal part of the perturbative expansion, based on a postulated ansatz for the skeleton expansion. We have shown that the ( $N_{f}$-independent) coefficients of this expansion and of the related BLM series have a precise interpretation when a perturbative infrared fixed point is present: they are the conformal coefficients in the series relating the fixed point value of the observable under consideration with that of the skeleton effective charge. The perturbative infrared fixed point appearing in multi-flavor QCD allows one to calculate these conformal coefficients through the Banks-Zaks expansion. We stress that the identification of the skeleton coefficients with the ones of the conformal relations defined in the small $\beta_{0}$ limit strongly relies on the particular ansatz we have taken, namely that the entire $N_{f}$ dependence originates in the running coupling itself, leaving the conformal coefficients $N_{f}$ independent. On the other hand, the identification of the BLM coefficients with those of the conformal relations of the small $\beta_{0}$ limit does not rely on any additional assumption, and it holds independently of the particular way BLM scale setting is performed.

The existence of an underlying skeleton structure implies that BLM (conformal) coefficients do not diverge factorially due to renormalons. Of course, there can be other effects 
which could make these coefficients diverge such as combinatorial factors related to the multiplicity of diagrams. Since in QCD this type of divergence is much softer than that of renormalons, we expect the BLM and possibly also the Banks-Zaks expansions to be "better behaved." This expectation is supported to some extent by previous observations concerning the smallness of the first few known BLM coefficients [12] and the Banks-Zaks coefficients [36-38]. On the other hand, the absence of renormalons does not always seem to be a sufficient explanation of the observed difference between conformal and non-conformal coefficients. At the same time, large coefficients which are not associated with running-coupling effects do appear in QCD, e.g. in conformal relations with the $\overline{\mathrm{MS}}$ coupling.

The uniqueness of the skeleton coupling in QED, which is identified as the Gell-Mann-Low effective charge, is an essential ingredient of the dressed skeleton expansion. It is still an open question whether an Abelian-like skeleton expansion exists in QCD and what the constraints are which would determine the skeleton coupling uniquely. The pinch technique may provide the answer [23-25] once it is systematically carried out to higher orders. We recall that the skeleton coupling is not constrained from the considerations raised in this paper: the only requirement following from the large $N_{f}$ limit is that $\bar{\beta}_{i}$ in this scheme does not contain an $N_{f}^{i+1}$ term. Since the decomposition of the coefficients (45) can be performed in any scheme yielding the moments $r_{i}^{(j)}$ to arbitrary high order, the corresponding functions $\phi_{i}$ can be formally constructed, up to the limitations discussed in Sec. III D. It thus seems that one can formally associate a "skeleton expansion" to any given coupling. The absence of renormalons in the conformal coefficients in a specific scheme implies that there are other schemes which share the same property: it is straightforward to see from the definition of the skeleton terms $R_{i}$ that an $N_{f}$-independent re-scaling of the argument of the coupling leaves the conformal coefficients unchanged. More generally, any "renormalon-free" transformation of the skeleton coupling would leave the "skeleton coefficients" free of renormalons. It is certainly interesting to find further constraints on the identity of the skeleton effective charge in QCD.

The BLM method provides a pragmatic way to deal with running-coupling effects beyond the single dressed gluon level. By decomposing the perturbative coefficients in the specific way implied by the skeleton expansion the contributions from the different skeleton integrals as well as the conformal coefficients can be identified. The BLM scales are then set such that there is a one-to-one correspondence between the terms in the BLM series and the skeletons, provided that BLM scale setting is performed in the skeleton scheme. As an alternative to the BLM scale setting procedure we saw that the skeleton integrals can also be approximated by applying the method of effective charges to the separate skeleton terms.

In practice, BLM scale setting can also be applied in physical schemes yielding a commensurate scale relation. This way conformal relations, which have a natural, maximally convergent, form (like the conformal Crewther rela- tion), can be used as a template for real-world QCD predictions, even if the underlying skeleton structure is not completely understood. Still, since the conformal relation between the fixed-point value of a generic observable and that of the skeleton effective charge is renormalon free, it follows, upon eliminating the skeleton effective charge, that the coefficients in commensurate scale relations between observables are also renormalon free. When such a conformal template is used for real-world QCD calculations, the effect of the non-zero $\beta$ function is to modify the values of the scales $k^{2}$ of the effective charge at each order of the expansion. We stress, however, that having no more correspondence with the skeleton expansion, the motivation for a particular scalesetting procedure is lost.

The BLM procedure cannot replace an eventual diagrammatic formulation of the skeleton expansion. We saw that the scale-setting prescription depends on the ansatz for the skeleton expansion, and any unknown concerning the form of the latter would have some impact on the former. We have considered several ways in which the simple ansatz we introduced, Eq. (2), may be generalized. This includes in particular the possibility that several skeleton diagrams will appear at the same order and that certain skeleton diagrams will contain some fermion loops as part of their structure, making the corresponding conformal coefficients $N_{f}$ dependent. In addition, we have seen that even in the case of a simple form of the skeleton expansion, the skeleton decomposition of the coefficients cannot be performed up to arbitrarily high order just based on the $N_{f}$ dependence, but rather requires some additional knowledge based on an explicit diagrammatic formulation. One should also be aware of the possibility that an Abelian-like skeleton expansion with a single effective charge might fail to exist in QCD. The non-Abelian skeleton expansion may then be based on several dressed Green functions, namely several different effective charges. Even in this more complicated case the most important properties of the skeleton expansion assumed here may hold. This includes the possibility to associate running-coupling effects to the various skeleton terms in a renormalization-group invariant way and the interpretation of the skeleton coefficients as conformal coefficients when a perturbative infrared fixed point is present.

\section{ACKNOWLEDGMENTS}

J.R. would like to thank the SLAC theory group for its generous hospitality during his visit last year. Center de Physique Théorique de l'Ecole Polytechnique is Laboratoire associe au CNRS UMR C7644. Laboratoire de Physique Théorique is Laboratoire associe au CNRS UMR 8627. This research was supported in part by the EC program "Training and Mobility of Researchers," Network "QCD and Particle Structure," contract ERBFMRXCT980194, and the U.S. Department of Energy, contract DE-AC03-76SF00515.

\section{APPENDIX: THE SKELETON EXPANSION AND THE EFFECTIVE CHARGE APPROACH}

A priori, the skeleton expansion approach, which relies on the assumption of a universal skeleton coupling, seems an- 
TABLE I. Comparison of effective charge $\beta$ function coefficients in the large $\beta_{0}$ approximation given by the width of $\phi_{0}$, $\beta_{2,3}=r_{2}^{(2)}-\left(r_{1}^{(1)}\right)^{2}$.

\begin{tabular}{ccccc}
\hline \hline $\bar{\beta}_{2,3}$ & $\beta_{2,3}^{D}$ & $\beta_{2,3}^{g_{1}}$ & $\beta_{2,3}^{F_{1}}$ & $\beta_{2,3}^{V}$ \\
\hline 0 & 2.625 & 2.389 & 1.500 & 0 \\
\hline \hline
\end{tabular}

tagonist to the original effective charge approach [5] which treats all effective charges independently and in a symmetric manner. In Sec. IV we saw how the ECH method can be used in the framework of the skeleton expansion to approximate separately each skeleton term $R_{i}$. Here we revisit the original ECH approach which attempts to evaluate the entire observable directly, and examine it from the point of view of the assumed skeleton expansion.

We begin by comparing the original ECH approach to the application of the ECH method for the leading skeleton term $R_{0}$. The first difference is, of course, in the ECH scale parameter. To facilitate the comparison, suppose that we start with a perturbative expansion (1) of the observable $a_{R}$ in terms of $\bar{a}\left(Q^{2}\right)$, with the corresponding coefficients $r_{i}$. In the original ECH approach this implies a scale ratio of $\Lambda_{R}^{2} / \bar{\Lambda}^{2}=e^{-r_{1} / \beta_{0}}$. This can be compared with Eq. (54). The difference between the two is due to the $r_{1}^{(0)}$ component in the next-to-leading coefficient $r_{1}$, the component which is not associated with the leading-skeleton. In practice, in many cases in QCD the running-coupling component dominates the next-to-leading coefficient. In such cases the two scales are close.

Next, also the $\beta$ function of the ECH method, $\beta_{R}\left(a_{R}\right)$ $\equiv d a_{R} / \ln Q^{2}$, is different (beyond the universal two-loop order) from that of $R_{0}$. At the three-loop level the latter is given in Eq. (56) whereas the former is

$$
\beta_{2}^{R}=\beta_{2,0}^{R}+\beta_{2,1}^{R} \beta_{0}+\beta_{2,2}^{R} \beta_{0}^{2}+\left[r_{2}^{(2)}-\left(r_{1}^{(1)}\right)^{2}\right] \beta_{0}^{3},
$$

where we exhibited the fact that the term leading in $\beta_{0}$ is the same in $\beta_{2}^{R_{0}}$ and $\beta_{2}^{R}$. As noted in Sec. IV, in the large $\beta_{0}$ limit $\beta_{2}^{R_{0}}$ is proportional to the width of the distribution $\phi_{0}$, namely to $\left[r_{2}^{(2)}-\left(r_{1}^{(1)}\right)^{2}\right]$. This remains correct also for the $\beta$ function of the full effective charge $a_{R}$ since adding subleading skeleton terms would not modify the leading $\mathcal{O}\left(\beta_{0}^{3}\right)$ term. For the four examples considered in Sec. VII, this parameter is given in Table I.

It is natural now to consider the possibility that $R_{0}$ is a good approximation to the observable $a_{R}$. In the effective charge approach at the next-to-next-to-leading order, this can be realized if $\beta_{2}^{R_{0}}$ is a good approximation to $\beta_{2}^{R}$. In the large $\beta_{0}$ limit the two are equal. Beyond the large $\beta_{0}$ limit one can ask whether

$$
\beta_{2,0}^{R}+\beta_{2,1}^{R} \beta_{0}+\beta_{2,2}^{R} \beta_{0}^{2} \simeq \bar{\beta}_{2} \equiv \bar{\beta}_{2,0}+\bar{\beta}_{2,1} \beta_{0}+\bar{\beta}_{2,2} \beta_{0}^{2},
$$

namely whether $\beta_{2,0}^{R}+\beta_{2,1}^{R} \beta_{0}+\beta_{2,2}^{R} \beta_{0}^{2}$ for a generic observable which admits a skeleton expansion, is approximately universal and close to the three-loop skeleton coupling $\beta$ function coefficient $\bar{\beta}_{2}$. If this holds for arbitrary $\beta_{0}$, then

$$
\beta_{2, i}^{R} \simeq \bar{\beta}_{2, i}
$$

for $i=0,1,2$. The violation of the equalities in Eqs. (A2) and (A3) is, of course, due to sub-leading terms in the skeleton expansion $R_{1}$ and $R_{2}$. This can be seen explicitly by substituting $r_{i}$ of Eq. (45) in the general relation

$$
\beta_{2}^{R}=\bar{\beta}_{2}+\beta_{0}\left(r_{2}-r_{1}^{2}\right)-\beta_{1} r_{1}
$$

to obtain the "skeleton decomposition" of $\beta_{2}^{R}$ :

$$
\begin{aligned}
\beta_{2}^{R}= & \bar{\beta}_{2}+\left(s_{2}-s_{1}^{2}\right) \beta_{0}+s_{1}\left(r_{2}^{(1)}-2 r_{1}^{(1)}\right) \beta_{0}^{2} \\
& +\left[r_{2}^{(2)}-\left(r_{1}^{(1)}\right)^{2}\right] \beta_{0}^{3}-s_{1} \beta_{1} .
\end{aligned}
$$

Finally, decomposing $\bar{\beta}_{2}$ and $\beta_{1}$ in terms of $\beta_{0}$, we obtain ${ }^{11}$

$$
\begin{aligned}
\beta_{2}^{R}= & {\left[\bar{\beta}_{2,0}-\beta_{1,0} s_{1}\right]+\left[\bar{\beta}_{2,1}-\beta_{1,1} s_{1}+\left(s_{2}-s_{1}^{2}\right)\right] \beta_{0} } \\
& +\left[\bar{\beta}_{2,2}+\left(r_{2}^{(1)}-2 r_{1}^{(1)}\right) s_{1}\right] \beta_{0}^{2}+\left[r_{2}^{(2)}-\left(r_{1}^{(1)}\right)^{2}\right] \beta_{0}^{3} .
\end{aligned}
$$

Clearly, if for a given observable the skeleton coefficients determining the normalization of the sub-leading skeleton terms $\left(s_{i}\right)$ are small, then even away from the large $\beta_{0}$ limit $\beta_{2}^{R}$ will be close to $\beta_{2}^{R_{0}}$.

In order to check Eq. (A3) explicitly for a given observable, one needs to calculate the $\beta$ function coefficients of both the observable effective charge $\beta_{2, i}^{R}$ and the skeleton effective charge $\bar{\beta}_{2, i}$. For the latter we currently know only $\bar{\beta}_{2,0}$ (see below) and so the examination of Eq. (A3) for $\bar{\beta}_{2,1}$ and $\bar{\beta}_{2,2}$ cannot yet be accomplished.

To obtain $\bar{\beta}_{2,0}$ we can use the general result [48] or, alternatively, use Eq. (A6), which is valid for a generic effective charge which admits a skeleton expansion. The latter yields

$$
\bar{\beta}_{2,0}=\beta_{2,0}^{R}+\beta_{1,0} s_{1} .
$$

Using this relation for various effective charges, e.g. the vacuum polarization $\mathrm{D}$ function (73) or the Bjorken sum rule (75), in the skeleton coupling scheme (12) defined through the pinch technique, we obtain

\footnotetext{
${ }^{11}$ The scheme of the skeleton coupling can be parametrized at the three-loop order [5] by the next-to-leading order coefficient $\left(s_{1}\right.$ and $r_{1}^{(1)}$ ) and by $\bar{\beta}_{2}$, i.e. $\bar{\beta}_{2, i}$ for $i=0,1,2$. Equation (A6) then shows explicitly that the effective charge $\beta$ function coefficient $\beta_{2}^{R}$ determines uniquely the remaining coefficients of the "skeleton decomposition" (45), namely, $s_{2}, r_{2}^{(1)}$ and $r_{2}^{(2)}$. This reflects the observation in Sec. III that, formally, the "skeleton decomposition" can be performed in any scheme.
} 
TABLE II. Comparison of effective charge $\beta$ function coefficients.

\begin{tabular}{ccrrrr}
\hline \hline$i$ & $\bar{\beta}_{2, i}$ & \multicolumn{1}{c}{$\beta_{2, i}^{D}$} & \multicolumn{1}{c}{$\beta_{2, i}^{g_{1}}$} & \multicolumn{1}{c}{$\beta_{2, i}^{F_{1}}$} & \multicolumn{1}{c}{$\beta_{2, i}^{V}$} \\
\hline 0 & -17.477 & -23.607 & -30.294 & -34.753 & -37.54 \\
1 & $?$ & -16.032 & -11.282 & -6.903 & 5.366 \\
2 & $?$ & 8.210 & 8.057 & 8.783 & 11.740 \\
\hline \hline
\end{tabular}

$$
\bar{\beta}_{2,0}=\frac{C_{A}}{512}\left(44 C_{F}^{2}-88 C_{A} C_{F}-301 C_{A}^{2}\right)
$$

and, for $N_{c}=3$,

$$
\bar{\beta}_{2,0}=-\frac{26845}{1536} \simeq-17.477 .
$$

Finally we check to what extent the suggested universality of the effective charge $\beta$ function coefficients (A3) holds for the four effective charges examined in Sec. VI, namely the effective charges related to the vacuum polarization D function (73) and the polarized, Eq. (75), and non-polarized, Eq. (76), Bjorken sum rules, as well as the static potential. The known coefficients are listed in Table II.

Although the coefficients $\beta_{2, i}^{R}$ for these observables have some common trend (e.g. for a given $i$ the signs are the same, with the exception of $\beta_{2, i}^{V}$ for $i=1$ ) it turns out that the fluctuations in their magnitude are rather large. In particular, in case of $\beta_{2,0}^{R}$ for which we know the value of the universal piece characterizing the skeleton coupling $\bar{\beta}_{2,0}$, the latter and the contribution of the sub-leading skeleton $R_{1}$ [through $s_{1}$ in Eq. (A6)] are of the same order of magnitude. The fluctuations between different observables are moderate only for $\beta_{2,2}^{R}$.

In [37] it has been observed that $\beta_{2}^{R}$ for the observables considered above (the static potential excluded) exhibit very close numerical proximity, especially for $N_{f}=0-7$. The extent to which universality of the sort examined here Eq. (A3) holds is not enough to explain this finding of [37].

The proximity of $\beta_{2,2}^{R}$ for the various effective charges implies that applying multi-scale BLM scale setting for one observable in terms of another, the second scale shift $t_{1,0}$ would be close to the leading skeleton scale shift $t_{0,0}$. In this case the single scale setting procedure $[8,18]$ could give similar results. The same holds in the skeleton scheme, if $\bar{\beta}_{2,2}$ is close to $\beta_{2,2}^{R}$. This can be deduced from Eq. (A6) which gives,

$$
\beta_{2,2}^{R}-\bar{\beta}_{2,2}=s_{1}\left(r_{2}^{(1)}-2 r_{1}^{(1)}\right)=2 s_{1}\left(t_{1,0}-t_{0,0}\right),
$$

where in the last step we used the leading order results for the scale shifts in Eqs. (26) and (29). In this respect it is interesting to note that applying the multi-scale BLM prescription in the $\overline{\mathrm{MS}}$ scheme, one in general obtains large values for the $t_{1,0}$ scale shift since $\beta_{2,2} \overline{\mathrm{MS}}=3.385$ is not close to $\beta_{2,2}$ of the physical effective charges. For example, when applying the BLM prescription to $a_{D}\left(a_{\overline{\mathrm{MS}}}\right)$ one obtains $k_{0,0}=0.707 Q$ and $k_{1,0}=0.366 \times 10^{-6} Q$. This can be contrasted, for instance, with the BLM scales for $a_{D}\left(a_{V}\right): k_{0,0}$ $=1.628 Q$ and $k_{1,0}=2.487 Q$.
[1] G. 't Hooft, in The Whys of Subnuclear Physics, Erice, 1977, edited by A. Zichichi (Plenum, New York, 1979), p. 94.

[2] A. H. Mueller, Nucl. Phys. B250, 327 (1985); Phys. Lett. B 308, 355 (1993).

[3] V. I. Zakharov, Nucl. Phys. B385, 452 (1992).

[4] For a recent review see M. Beneke, Phys. Rep. 317, 1 (1999).

[5] G. Grunberg, Phys. Lett. 95B, 70 (1980); 110B, 501(E) (1982); Phys. Rev. D 29, 2315 (1984).

[6] P. M. Stevenson, Phys. Rev. D 23, 2916 (1981).

[7] S. J. Brodsky, G. P. Lepage, and P. B. Mackenzie, Phys. Rev. D 28, 228 (1983); G. P. Lepage and P. B. Mackenzie, ibid. 48, 2250 (1993).

[8] G. Grunberg and A. L. Kataev, Phys. Lett. B 279, 352 (1992).

[9] S. J. Brodsky and J. Rathsman, hep-ph/9906339.

[10] H. J. Lu and C. A. R. Sa de Melo, Phys. Lett. B 273, 260 (1991); 285, 399(E) (1992); H. J. Lu, Phys. Rev. D 45, 1217 (1992); Ph.D. thesis, Stanford, 1992, SLAC-0406.

[11] C. P. Lepage and P. B. Mackenzie, Phys. Rev. D 48, 2250 (1993).

[12] S. J. Brodsky and H. J. Lu, Phys. Rev. D 51, 3652 (1995).

[13] J. Rathsman, Phys. Rev. D 54, 3420 (1996).

[14] M. Neubert, Phys. Rev. D 51, 5924 (1995).

[15] M. Beneke and V. M. Braun, Phys. Lett. B 348, 513 (1995); P. Ball, M. Beneke, and V. M. Braun, Nucl. Phys. B452, 563 (1995).
[16] C. N. Lovett-Turner and C. J. Maxwell, Nucl. Phys. B432, 147 (1994).

[17] S. J. Brodsky, J. Ellis, E. Gardi, M. Karliner, and M. A. Samuel, Phys. Rev. D 56, 6980 (1997).

[18] S. J. Brodsky, G. T. Gabadadze, A. L. Kataev, and H. J. Lu, Phys. Lett. B 372, 133 (1996).

[19] S. J. Brodsky, M. Melles, and J. Rathsman, Phys. Rev. D 60, 096006 (1999)

[20] S. J. Brodsky, M. S. Gill, M. Melles, and J. Rathsman, Phys. Rev. D 58, 116006 (1998).

[21] J. D. Bjorken and S. D. Drell, Relativistic Quantum Fields (McGraw-Hill, New York, 1965).

[22] M. Baker and C. Lee, Phys. Rev. D 15, 2201 (1977); 17, 2182(E) (1978).

[23] J. M. Cornwall, Phys. Rev. D 26, 1453 (1982); J. M. Cornwall and J. Papavassiliou, ibid. 40, 3474 (1989); ibid. 47, 4728 (1993); 41, 3179 (1990); G. Degrassi and A. Sirlin, ibid. 46, 3104 (1992).

[24] N. J. Watson, Nucl. Phys. B494, 388 (1997); B552, 461 (1999).

[25] J. Papavassiliou, Phys. Rev. D 62, 045006 (2000); Phys. Rev. Lett. 84, 2782 (2000).

[26] T. Banks and A. Zaks, Nucl. Phys. B196, 189 (1982).

[27] G. Grunberg, Phys. Rev. D 46, 2228 (1992). 
[28] A. R. White, Phys. Rev. D 29, 1435 (1984); Int. J. Mod. Phys. A 8, 4755 (1993).

[29] Yu. L. Dokshitzer, G. Marchesini, and B. R. Webber, Nucl. Phys. B469, 93 (1996).

[30] G. Grunberg, J. High Energy Phys. 11, 006 (1998).

[31] E. Gardi and G. Grunberg, J. High Energy Phys. 11, 016 (1999).

[32] R. J. Crewther, Phys. Rev. Lett. 28, 1421 (1972); Phys. Lett. B 397, 137 (1997).

[33] D. J. Broadhurst and A. Kataev, Phys. Lett. B 315, 179 (1993).

[34] D. J. Gross and F. Wilczek, Phys. Rev. D 8, 3633 (1973).

[35] W. E. Caswell, Phys. Rev. Lett. 33, 244 (1974).

[36] S. A. Caveny and P. M. Stevenson, hep-ph/9705319.

[37] E. Gardi and M. Karliner, Nucl. Phys. B529, 383 (1998).

[38] E. Gardi and G. Grunberg, J. High Energy Phys. 03, 024 (1999).

[39] S. J. Brodsky and P. Huet, Phys. Lett. B 417, 145 (1998).
[40] D. Gross and F. Wilczek, Phys. Rev. Lett. 30, 1343 (1973); H. D. Politzer, ibid. 30, 1346 (1973).

[41] D. J. Broadhurst and A. G. Grozin, Phys. Rev. D 52, 4082 (1995).

[42] C. J. Maxwell and A. Mirjalili, Nucl. Phys. B577, 209 (2000).

[43] S. G. Gorishny, A. L. Kataev, and S. A. Larin, Phys. Lett. B 259, 144 (1991); L. R. Surguladze and M. A. Samuel, Phys. Rev. Lett. 66, 560 (1991); 66, 2416(E) (1991).

[44] S. A. Larin and J. A. M. Vermaseren, Phys. Lett. B 259, 345 (1991).

[45] S. A. Larin, F. V. Tkachov, and J. A. M. Vermaseren, Phys. Rev. Lett. 66, 862 (1991).

[46] M. Peter, Phys. Rev. Lett. 78, 602 (1997); Nucl. Phys. B501, 471 (1997); Y. Schröder, Phys. Lett. B 447, 321 (1999).

[47] J. Ellis, E. Gardi, M. Karliner, and M. A. Samuel, Phys. Lett. B 366, 268 (1996).

[48] G. Grunberg, Phys. Lett. 135B, 455 (1984). 